\title{
Reconstructing Nonequilibrium Regimes of Quantum Many-Body Systems from the Analytical Structure of Perturbative Expansions
}

\author{
Corentin Bertrand $\odot,{ }^{1}$ Serge Florens, ${ }^{2}$ Olivier Parcollet, ${ }^{3,4}$ and Xavier Waintal ${ }^{1}$ \\ ${ }^{1}$ Université Grenoble Alpes, CEA, INAC-PHELIQS, GT F-38000 Grenoble, France \\ ${ }^{2}$ Université Grenoble Alpes, CNRS, Institut Néel, F-38000 Grenoble, France \\ ${ }^{3}$ Center for Computational Quantum Physics, Flatiron Institute, 162 th Avenue, \\ New York, New York 10010, USA \\ ${ }^{4}$ Institut de Physique Théorique (IPhT), CEA, CNRS, UMR 3681, 91191 Gif-sur-Yvette, France
}

(Received 29 March 2019; revised manuscript received 7 June 2019; published 8 October 2019)

\begin{abstract}
We propose a systematic approach to the nonequilibrium dynamics of strongly interacting many-body quantum systems, building upon the standard perturbative expansion in the Coulomb interaction. Highorder series are derived from the Keldysh version of the determinantal diagrammatic quantum Monte Carlo algorithm, and the reconstruction beyond the weak-coupling regime of physical quantities is obtained by considering them as analytic functions of a complex-valued interaction $U$. Our advances rely on two crucial ingredients: (i) a conformal change of variable, based on the approximate location of the singularities of these functions in the complex $U$ plane, and (ii) a Bayesian inference technique, that takes into account additional known nonperturbative relations, in order to control the amplification of noise occurring at large $U$. This general methodology is applied to the strongly correlated Anderson quantum impurity model and is thoroughly tested both in and out of equilibrium. In the situation of a finite voltage bias, our method is able to extend previous studies, by bridging with the regime of unitary conductance and by dealing with energy offsets from particle-hole symmetry. We also confirm the existence of a voltage splitting of the impurity density of states and find that it is tied to a nontrivial behavior of the nonequilibrium distribution function. Beyond impurity problems, our approach could be directly applied to Hubbard-like models, as well as other types of expansions.
\end{abstract}

DOI: 10.1103/PhysRevX.9.041008

Subject Areas: Condensed Matter Physics,

Mesoscopics,

Strongly Correlated Materials

\section{INTRODUCTION}

The study of the out-of-equilibrium regime of strongly correlated many-body quantum problems is a major challenge in theoretical condensed matter physics. Its interest has grown rapidly in the past few years with new experiments, e.g., the ability to control light-matter interaction on an ultrafast timescale [1], light-induced superconductivity [2-6], or a metal-insulator transition driven by an electric field [7], proposed, e.g., to build artificial neurons [8]. These experiments raise the question whether the combination of strong correlation effects and out-of-equilibrium regimes could lead to genuinely new physics and phases of matter that do not have an equilibrium counterpart. Quantum nanoelectronics also provide many examples of

Published by the American Physical Society under the terms of the Creative Commons Attribution 4.0 International license. Further distribution of this work must maintain attribution to the author(s) and the published article's title, journal citation, and DOI. such systems. A classic example is the spin-1/2 Kondo effect occurring in a quantum dot, but recent experiments have also managed to study in great detail underscreened [9,10] and overscreened [11,12] (multichannel) Kondo effects, characterized by non-Fermi-liquid fixed points. Other notable examples of new quantum states induced by interactions are Luttinger liquids [13] that take place at edges in the fractional quantum Hall regime or the " 0.7 anomaly" [14-16] occurring in a simple quantum point contact geometry. Last, solid-state-based quantum computers such as spin qubit devices are nothing but out-ofequilibrium quantum many-body systems (few site Hubbard-like models, possibly connected to electrodes) that bring new questions into the scope of correlated systems [17].

It is worth noting that even the simplest of these outof-equilibrium problems, the single-impurity Anderson model, is still the subject of active research $[18,19]$. Early approaches use a range of approximate techniques including fourth-order perturbation theory [20], equation of motion techniques [21], and the noncrossing approximation (NCA) [22]. State-of-the-art techniques include the timedependent numerical renormalization group (NRG) and the 
density matrix renormalization group [19,23-28]. Early attempts of a real-time quantum Monte Carlo algorithm [29-33] experienced an exponential sign problem at a long time and large interaction. Within Monte Carlo methods, two main routes are currently explored to resolve this issue: the inchworm algorithm [34-38] and the SchwingerKeldysh diagrammatic quantum Monte Carlo (QMC) [39] algorithm. The latter, which we use in this paper, reaches the infinite time steady-state limit and has a complexity which does not grow with time. The development of controlled computational methods is critical for the development of the theory in this field. Beyond its direct application to impurities and quantum dot physics, the Anderson model is of direct interest for quantum embedding methods such as the dynamical mean field theory (DMFT) [40-42] which reduce the bulk lattice problem to the solution of a self-consistent quantum impurity model.

A straightforward approach to study the out-ofequilibrium many-body quantum problem is to compute the systematic perturbative expansion of some physical quantity $F$ in power of the electron-electron interaction $U$ : $F(U) \equiv \sum_{n=0}^{\infty} F_{n} U^{n}$. In practice, $F$ may depend on the time (or frequency) as well as the voltage bias, temperature, etc. The coefficients $F_{n}$ are given by the out-of-equilibrium Schwinger-Keldysh version of the Feynman diagrams [43]. Such a perturbative expansion is a central tool in quantum mechanics and quantum field theory. In weak-coupling theories, a few orders are sufficient to explain many physical phenomena, even quantitatively, as, e.g., in quantum electrodynamics. However, at intermediate or strong coupling, this approach faces two main challenges: (i) the computation of the coefficients for $n$ large enough and (ii) the reconstruction of the physical quantities as a function of $U$ from a finite number of coefficients.

Using the standard Wick theorem, an explicit expression of $F_{n}$ to order $n$ can be written as $n$-dimensional integrals. While the computation of $F_{n}$ can hardly be achieved analytically beyond a few orders, QMC algorithms known as "diagrammatic Monte Carlo" [44-59] are able to compute a finite number of these coefficients $F_{n}$ for a general class of quantum many-body problems, in practice up to 8 or 15 depending on the model and the physical quantity. The first generation of these algorithms used to explicitly sample the Feynman diagrams one by one with a complex Markov chain, moving from one diagram to another. A second generation of algorithms handles the diagrams collectively using combinations of determinants to cancel disconnected diagrams in physical quantities. This rewriting was achieved in the real-time SchwingerKeldysh formalism [39] and in the imaginary-time Matsubara formalism [60-63].

The resummation of the series is a nontrivial mathematical task outside of the weak-coupling regime, even with a perfect knowledge of the coefficients $F_{n}$. The issue comes from the finite radius of convergence of the series.
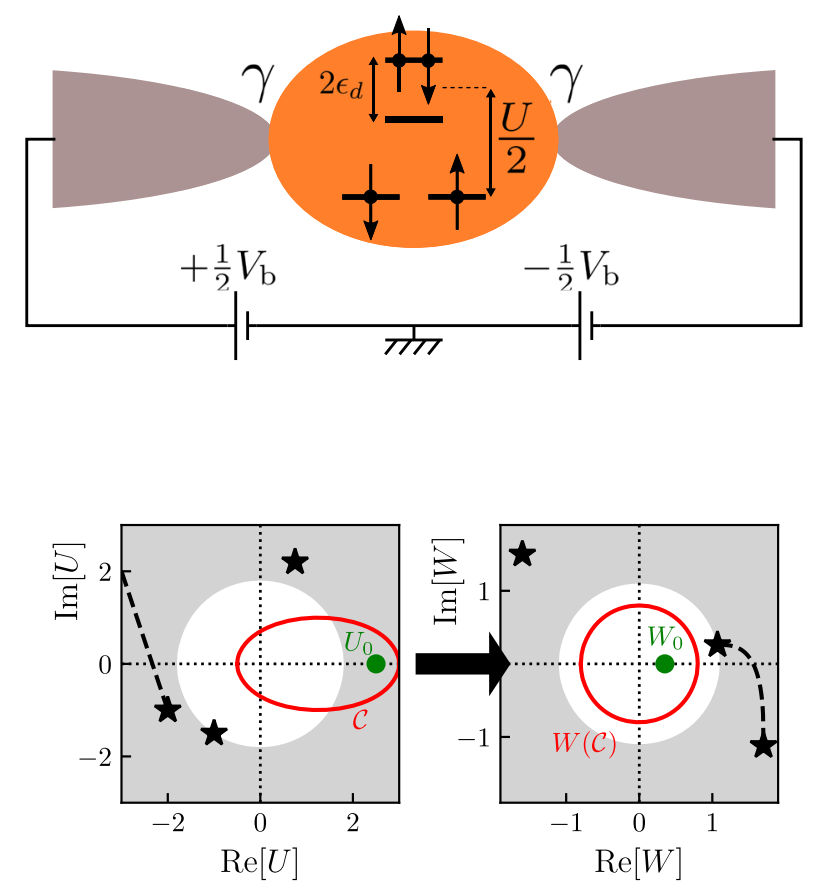

FIG. 1. Top: The Anderson quantum impurity model describing a single-level quantum dot. The level with energy $\epsilon_{d}$ is subject to a finite Coulomb interaction $U$ and is hybridized with a tunnel coupling $\gamma$ to two leads that are biased with voltage $V_{b}$. Bottom: Illustration of the general computation scheme developed in this work. A physical quantity $F$ (e.g., the current through the dot) presents singularities in the $U$ complex plane (left), such as poles (stars) or branch cuts (dashed line), hampering the proper convergence of perturbative approaches for values of $U$ outside the convergence disk (gray area). After defining a broad singularity-free contour $\mathcal{C}$ (red line) that encircles both $U=0$ and a targeted $U_{0}$ value, a conformal map $U \rightarrow W(U)$ is defined in order to bring $W_{0}=W\left(U_{0}\right)$ inside the convergence disk of $F[U(W)]$ (right). Resummation techniques can then be applied in a controlled way.

When $U$ is larger than this radius, the truncated series to the first $N$ th terms does not converge with $N$ and some resummation technique must be used to compute $F(U)$. Moreover, there are two additional difficulties associated with numerical methods: (i) Only a finite number of coefficients $F_{n}$ can be computed, since the computation cost is exponential in $n$, and (ii) the $F_{n}$ are known only with a finite precision, typically of a few digits in QMC.

In this paper, we approach this problem from the angle of complex analysis. Indeed, the divergence of the series originates from the singularity structure of the function $F(U)$ in the complex plane $U$ (bottom left in Fig. 1). We discuss how to locate the singularities closest to 0 and how to construct an analytic change of variable to resum the series beyond weak coupling (bottom right in Fig. 1). We also introduce a Bayesian technique to take into account the amplification of the Monte Carlo noise in the resummation process using some simple nonperturbative additional information on the model. 
While our approach is quite general, we focus here on the nonequilibrium Anderson quantum impurity model in the quantum dot configuration (top in Fig. 1). Our starting point is an expansion of the Green's function in power of the Hubbard interaction $U$, using an extension of the algorithm of Ref. [39]. The algorithm is discussed in detail in a companion paper [64], and its implementation is based on the TRIQS library [65]. This algorithm provides a numerically exact computation of the perturbative series of physical quantities in power of the interaction $U$, at a cost which is uniform in time but exponential with the expansion order. Hence, it allows one to compute in a transient regime as well as directly in a long time steady state, a regime in which most competing methods have severe limitations.

This paper is organized as follows. Section II introduces our notations for the single-impurity Anderson model. Section III develops the resummation technique and illustrates it on the Kondo temperature. Section IV performs a benchmark of the method against the NRG for the equilibrium dynamics. Section V presents new results in the nonequilibrium regime, including the voltage-split spectral function, extended-range current-voltage characteristics, and a nontrivial dot distribution function. Section VI concludes this article and presents perspectives for our conformal approach to the perturbative expansions of strongly interacting quantum systems.

\section{THE ANDERSON IMPURITY MODEL}

In this paper, we focus on the single-impurity Anderson model both at and out of equilibrium. While originally formulated to describe the effect of magnetic impurities in metals, this model is widely used in theoretical condensed matter, both as a simple model for quantum dots in mesoscopic physics and as a building block of "quantum embedding" approximations like DMFT and its generalizations. At the core of the Anderson model lies Kondo physics. The repulsive interaction on the quantum dot leads to an effective antiferromagnetic interaction between the electronic reservoirs and the spin of the (unique) electron trapped in the quantum dot in the local moment regime. This interaction leads to the formation of the Kondo resonance, a thin peak in the local density of state pinned at the Fermi energy [66]. The Anderson impurity Hamiltonian reads

$$
\begin{aligned}
\hat{\mathbf{H}}= & \sum_{i=-\infty}^{+\infty} \sum_{\sigma} \gamma_{i} \hat{\mathbf{c}}_{i, \sigma}^{\dagger} \hat{\mathbf{c}}_{i+1, \sigma}+\text { H.c. }+\epsilon_{d}\left(\hat{\mathbf{n}}_{\uparrow}+\hat{\mathbf{n}}_{\downarrow}\right) \\
& +U \theta(t)\left(\hat{\mathbf{n}}_{\uparrow}-\frac{1}{2}\right)\left(\hat{\mathbf{n}}_{\downarrow}-\frac{1}{2}\right) .
\end{aligned}
$$

It connects an impurity on site 0 to two semi-infinite electrodes $i<0$ and $i>0$. The model corresponds to a single-level artificial atom as sketched in the upper panel in
Fig. 1. Here, $\epsilon_{d}$ is the on-site energy of the impurity (relative to the particle-hole symmetric point), and $\hat{\mathbf{n}}_{\sigma}=$ $\hat{\mathbf{c}}_{0, \sigma}^{\dagger} \hat{\mathbf{c}}_{0, \sigma}$ is the impurity density of spin $\sigma$ electrons. $\hat{\mathbf{c}}_{i, \sigma}^{\dagger}$ and $\hat{\mathbf{c}}_{i, \sigma}$ are the creation and annihilation operators, respectively, for electrons on site $i$ with spin $\sigma$. We use $\hbar=e=1 . \theta(t)$ is the Heaviside function: We switch the interaction on at time $t=0$. Typical calculations are performed for large times so that the system relaxes to its stationary regime. The hopping parameters are given by $\gamma_{i}=1$ except for $\gamma_{0}=$ $\gamma_{-1}=\gamma$ which connect the impurity to the electrodes. The calculations can be performed for arbitrary values of $\gamma$. However, since we are not interested in the large-energy physics of the electrodes, we suppose that $\gamma \ll 1$, i.e., that the tunneling rate from the impurity to the electrodes is energy-independent $\Gamma=2 \pi \gamma^{2} \rho_{F}$, where $\rho_{F}$ is the density of states of the electron reservoirs at the Fermi level. The noninteracting retarded Green's function of the free impurity is given by

$$
g^{R}(\omega)=\frac{1}{\omega-\epsilon_{d}+i \Gamma} .
$$

The two electrodes have a chemical potential symmetric with respect to zero $\pm V_{b} / 2$ which corresponds to a bias voltage $V_{b}$. They share the same temperature that we take very low: $T=10^{-4} \Gamma$. Within the standard nonequilibrium Keldysh formalism [67], the noninteracting lesser and greater Green's functions are given, respectively, by

$$
\begin{gathered}
g^{<}(\omega)=\frac{i \Gamma\left[n_{F}\left(\omega+\frac{V_{b}}{2}\right)+n_{F}\left(\omega-\frac{V_{b}}{2}\right)\right]}{\left(\omega-\epsilon_{d}\right)^{2}+\Gamma^{2}}, \\
g^{>}(\omega)=\frac{i \Gamma\left[n_{F}\left(\omega+\frac{V_{b}}{2}\right)+n_{F}\left(\omega-\frac{V_{b}}{2}\right)-2\right]}{\left(\omega-\epsilon_{d}\right)^{2}+\Gamma^{2}},
\end{gathered}
$$

where $n_{F}(\omega)=1 /\left(e^{\omega / T}+1\right)$ is the Fermi function. $g^{>}(\omega)$ and $g^{<}(\omega)$ are the starting point for the expansion in power of $U$ that will be performed with the real-time diagrammatic quantum Monte-Carlo method.

The quantities of interest in this article are the interacting Green's functions (denoted with capital letters)

$$
\begin{gathered}
G^{R}\left(t, t^{\prime}\right)=-i \theta\left(t-t^{\prime}\right)\left\langle\left\{\hat{\mathbf{c}}_{0 \uparrow}(t), \hat{\mathbf{c}}_{0 \uparrow}^{\dagger}\left(t^{\prime}\right)\right\}\right\rangle, \\
G^{<}\left(t, t^{\prime}\right)=i\left\langle\hat{\mathbf{c}}_{0 \uparrow}^{\dagger}\left(t^{\prime}\right) \hat{\mathbf{c}}_{0 \uparrow}(t)\right\rangle, \\
G^{>}\left(t, t^{\prime}\right)=-i\left\langle\hat{\mathbf{c}}_{0 \uparrow}(t) \hat{\mathbf{c}}_{0 \uparrow}^{\dagger}\left(t^{\prime}\right)\right\rangle,
\end{gathered}
$$

where the operators have been written in the Heisenberg representation. Since we restrict ourselves to the stationary limit, these functions are a function of $t-t^{\prime}$ only and can be studied in the frequency domain. Of particular interest is the spectral function (or interacting local density of state) given by 

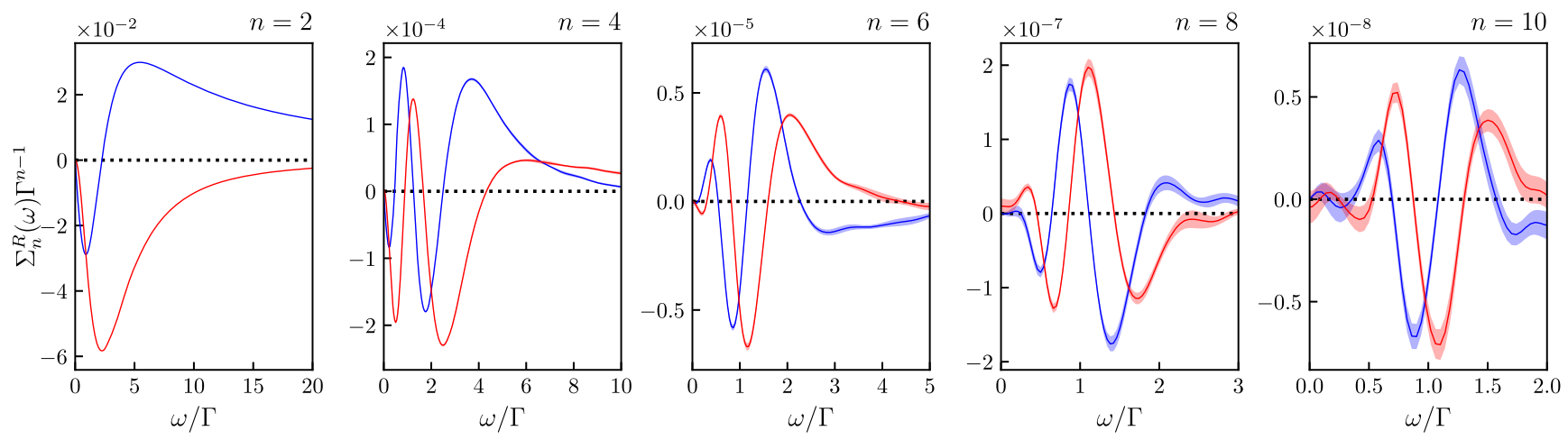

FIG. 2. First nonzero orders of the self-energy series $\Sigma^{R}(U, \omega)$ in powers of $U$ for the equilibrium particle-hole symmetric Anderson model (real part in blue and imaginary part in red). This series is computed with a real-time diagrammatic quantum Monte Carlo algorithm detailed in a companion article [64]. The statistical error is shown as shaded areas. Because of particle-hole symmetry, odd orders are zeros.

$$
A(\omega)=-\frac{1}{\pi} \operatorname{Im}\left[G^{R}(\omega)\right]
$$

The equilibrium spectral function displays the important features of Kondo physics: a sharp Kondo resonance at the Fermi level and satellite peaks around $\omega= \pm U / 2$ in the case of particle-hole symmetry.

The out-of-equilibrium spectral function can be used for the computation of the current-voltage characteristic using the Wingreen-Meir formula [68]:

$I=\frac{\Gamma}{2} \int A(\omega)\left[n_{F}\left(\omega+\frac{V_{b}}{2}\right)-n_{F}\left(\omega-\frac{V_{b}}{2}\right)\right] d \omega$.

The retarded self-energy $\Sigma^{R}(\omega)$ is defined from the interacting Green's function by

$$
G^{R}(\omega)=\frac{1}{\omega-\epsilon_{d}+i \Gamma-\Sigma^{R}(\omega)} .
$$

Physical quantities have a systematic expansion in power of $U$ :

$$
G^{R}\left(t-t^{\prime}\right)=\sum_{n=0}^{+\infty} G_{n}^{R}\left(t-t^{\prime}\right) U^{n}
$$

from which we obtain the corresponding quantity in the frequency domain by Fourier transform:

$$
G^{R}(\omega)=\sum_{n=0}^{+\infty} G_{n}^{R}(\omega) U^{n}
$$

We obtain the functions $G_{n}^{R}(\omega)$ (typically up to $n=10$ ) using the QMC algorithm of Refs. [39,64]. The expansion of the self-energy

$$
\Sigma^{R}(\omega)=\sum_{n=0}^{+\infty} \Sigma_{n}^{R}(\omega) U^{n}
$$

is obtained from the $G_{n}^{R}(\omega)$ using a formal series expansion order by order of the Dyson equation (8). As an illustration, Fig. 2 shows the self-energy series, up to the 10th order, for the equilibrium particle-hole symmetric model as obtained from diagrammatic QMC [64]. These series are the starting point of this paper, which is devoted to the resummation of the perturbative expansion for the Green's function and the self-energy beyond weak coupling.

\section{THE PERTURBATIVE SERIES BEYOND THE WEAK-COUPLING REGIME}

The diagrammatic quantum Monte Carlo algorithm yields the first orders of the perturbation expansion of physical quantities, with some error bars. In weak coupling, we can directly sum this series and obtain the physical quantities with a few orders. Beyond weak coupling, however, we face a more complex problem. For a given physical quantity $F$, we want to evaluate $F(U)$ from the first $N$ (typically $N \sim 10$ ) coefficients $F_{0}, F_{1}, F_{2} \ldots F_{N}$ of a series $F(U) \equiv \sum_{n=0}^{\infty} F_{n} U^{n}$. In the following, $F$ stands for the width of the Kondo peak, the Green's function $G$, or the self-energy $\Sigma$ of the impurity. In the latter cases, the coefficients are functions of the frequencies, $G_{n}(\omega)$ and $\Sigma_{n}(\omega)$. We also want to know, for a given physical quantity $F$ and interaction $U$, how many orders $N_{0}$ are needed to obtain $F(U)$ at a given precision. Since the cost of the diagrammatic QMC approach is exponential in $N_{0}$, the answer to this question gives the ultimate limit of the method.

The mathematical problem of series resummation is a quite old topic, e.g., Ref. [69]. Various techniques have been used in physics problems including Padé approximants [70], Lindelöf extrapolation [52,71], or the CesàroRiesz technique [48]. In diagrammatic QMC, this is 
typically (except in bold diagrammatic QMC) a postprocessing step: The Monte Carlo algorithm produces the values of the various orders of the expansion, and one then attempts to sum the series to obtain the final result. However, the situation is quite different if we want to use such a technique to solve quantum impurity models in the context of the quantum embedding methods like DMFT [40] or, e.g., Trilex [72,73]. Indeed, in such cases, the method requires multiple solutions of the impurity model to solve their self-consistency loop. Therefore, it is necessary to develop more robust methods to sum the perturbative series for impurity systems, which could be automatized.

In the cases considered in this paper (quantum impurity models), and in general for lattice models at a finite temperature (such as the Hubbard model), the series for $F$ is expected to have a nonzero radius of convergence $R_{F}$. Note that $R_{F}$ not only depends on the chosen physical quantity $F$, but may also depend on the frequency, voltage, temperature, etc. $R_{F}$ separates the weak-coupling regime $\left(|U|<R_{F}\right)$ from the strong-coupling regime $\left(|U|>R_{F}\right)$. At weak coupling, the truncated series $\sum_{n=0}^{N} F_{n} U^{n}$ provides an accurate estimate of $F(U)$ and is controlled exponentially with the number of coefficients $N$ [like a geometric series since $\left.F_{n} \sim\left(1 / R_{F}\right)^{n}\right]$. At strong coupling, however, this truncated series diverges. Note that in some problems like, e.g., the unitary fermionic gas, the series has a zero radius of convergence; see, e.g., Ref. [74] for a recent example with diagrammatic QMC. We do not consider these cases in this paper, as they require other techniques than the ones presented here, e.g., Borel summation techniques.

In this paper, we consider the series summation problem with the angle of reconstructing the function $F(U)$ in the complex $U$ plane. The divergence of the series is due to the presence of singularities in the complex $U$ plane, starting on the circle $|U|=R_{F}$. The question is to reconstruct $F$ beyond the radius of convergence.

\section{A. General theory}

\section{Conformal transformation}

Conformal transformations can be used to deform the complex plane and bring the point to be computed back into the convergence disk of a transformed series. This technique was used a long time ago, e.g., in statistical physics [75]. In a previous work [39], some of us showed that a simple conformal Euler transform allows one to compute the density on the impurity up to $U=\infty$, at a very low temperature, from the first 12 coefficients of the series. However, this Euler transform is not always successful in resumming other quantities like the Green's function and the self-energy and needs to be generalized.

Suppose that we aim at evaluating $F(U)$ at $U=U_{0}$ with $U_{0}$ real, positive, and $U_{0}>R_{F}$. First, we assume a separation property, i.e., that we can find a simply connected domain delimited by a curve $\mathcal{C}$ containing 0 and $U_{0}$ but no singularities of the function $F$, as illustrated in the bottom left of Fig. 1. The singularities of the function $F(U)$ are located outside the domain $\mathcal{C}$. We then proceed as follows.

(i) First, according to the Riemann mapping theorem, we can construct a biholomorphic change of variable $W(U)$ such that (i) $W(0)=0$ and (ii) it maps the interior of $\mathcal{C}$ into a disk $D_{\mathcal{C}}$ centered at 0 in the $W$ plane (see the bottom right of Fig. 1). In practice, we seek $\mathcal{C}$ to separate the singularities from the half straight line of real positive $U$. In the following, we use two simple transformations, but, in general, we could use a Schwarz-Christoffel map if $\mathcal{C}$ is a polygon [76], composed with a Möbius transformation of the disk to enforce (i).

(ii) Second, we form the series for the reciprocal function $U[W]$ of $W(U)$ which is defined term by term by the equation $U[W(U)]=U$. We then construct the series $\bar{F}(W) \equiv \sum_{p} \bar{F}_{p} W^{p}$ defined by the composition $\bar{F}(W)=F(U[W])$. Since $W(0)=0$, the first $N$ terms of $F(W)$ can be computed from the first $N$ terms of $F(U)$.

(iii) We evaluate the series $\bar{F}\left(W_{0}\right)$ at the point of interest $W_{0}=W\left(U_{0}\right)$. Indeed, by construction $W_{0} \in D_{\mathcal{C}}$, and, since $\bar{F}(W)$ is holomorphic in $D_{\mathcal{C}}, D_{\mathcal{C}}$ is included in the convergence disk of the series $\bar{F}$. Hence, the series $\bar{F}$ converges at $W_{0}$.

The result is independent of the choice of the domain $\mathcal{C}$, but the speed of convergence of the series for $\bar{F}\left(W_{0}\right)$ versus $N$ is not, since it is determined by the relative position of $W_{0}$ compared to the radius of convergence $R_{\bar{F}}$ of $\bar{F}$, i.e., $\eta_{\mathcal{C}} \equiv\left|W_{0} / R_{\bar{F}}\right|$. Therefore, there are ways to optimize the domain $\mathcal{C}$. For example, we cannot simply take a narrow domain close to the real axis, for the convergence in $W$ would be really slow: We need to have $U_{0}$ and 0 as "far" as possible from the curve $\mathcal{C}$ (the precise meaning of far being given by $\eta_{\mathcal{C}}$ ). For each domain $\mathcal{C}$ satisfying the separation property, there is a minimum number of orders $N_{\mathcal{C}}$ needed to obtain the result at a given precision $\epsilon$. There is, therefore, an optimal domain, which minimizes $N_{\mathcal{C}}$ to $N_{\text {opt }}=\min _{\mathcal{C}} N_{\mathcal{C}}$. This optimum is the absolute minimum of orders needed to sum the series and, therefore, determine in fine the complexity of the diagrammatic QMC algorithm. Our next goal is to approach such an optimum.

Note that a failure of the separation assumption, i.e., the choice of a domain containing singularities, may result simply in the divergence of the series $\bar{F}$ at $W_{0}$, and, hence, a clear failure of the method rather than a wrong result. Conversely, the study of the convergence radius of the $\bar{F}(W)$ series provides direct information on the singularityfree regions of the $U$ plane. Indeed, the region of the $U$ plane that is mapped into the inside of the convergence radius of $\bar{F}(W)$ are singularity- and branch-cut-free. Hence, using several conformal transforms, one may perform a step-by-step construction of the domain $\mathcal{C}$. Another note is that, as a consistency check, one can also check the stability of the final result upon small deformations of the domain 
[or the $W(U)$ function], as discussed in detail in Ref. [39] for the Euler transform.

The existence of the domain $\mathcal{C}$ and the transformation $W(U)$ has a direct consequence on the algorithmic complexity of the diagrammatic quantum Monte Carlo algorithm. It is shown in Ref. [77] that, for values of $U$ inside the convergence radius, connected diagrammatic quantum Monte Carlo techniques provide a systematic route for calculating the many-body quantum problem in a computational time that increases only polynomially with the requested precision. The result also applies to the Keldysh diagrammatic QMC. For completeness, the core of the argument is as follows: Inside the radius of convergence $R$, the precision of a calculation $\epsilon$ increases exponentially with the number of orders $N$ used $\epsilon \sim(U / R)^{N}$. Hence, although the computational time $C$ increases exponentially with $N, C \sim a^{N}$, the overall computational time scales as $C \sim(1 / \epsilon)^{\log a / \log (R / U)}$, i.e., polynomially; see Ref. [77] for a detailed analysis. For a given $U_{0}$ and domain $\mathcal{C}$, we now have to sum the transformed series $\bar{F}$ inside the radius of convergence. Hence, the same argument also applies for this series, and, therefore, we conclude that, even outside the disk of convergence, we expect the algorithm to have a polynomial complexity as a function of the precision. Let us emphasize, however, that this result is largely academic, since in practice the power law can be large. Moreover, as we discuss, for some physical quantities the transformation to $W$ can lead to a dramatic increase of the noise which induces a large computation time for a given precision.

\section{Location of singularities in the complex $U$ plane}

In order to choose $\mathcal{C}$ properly, we need to have some information on the location of the singularities in the $U$ plane. In this paper, we use the following technique to approximately locate the poles of $F(U)$ in the complex plane.

(i) We form an inverse of $F$ of the form $K(U)=$ $1 /[F(U)+a]$ as a formal series (i.e., order by order). $a$ is a constant that we choose at our convenience. In order for the series $K(U)$ to exist, we must have $F_{0}+a \neq 0$.

(ii) We estimate the radii of convergence $R_{F}$ (respectively, $R_{K}$ ) of $F$ (respectively, $K$ ), by plotting $\left|F_{n}\right|$ and $\left|K_{n}\right|$ versus $n$ and fitting the asymptote $\left|F_{n}\right| \sim\left(1 / R_{F}\right)^{n}$.

(iii) In most situations, we find $R_{F} \neq R_{K}$. If not, we use a different $a$ so as to obtain $R_{F} \neq R_{K}$. Without loss of generality, let us assume that $R_{K}$ is the largest. We use the truncated polynomial of the series, $\sum_{p=0}^{N} K_{p} U^{p}$, to compute $K(U)$ within its disk of convergence and, therefore, locate its zeros, which are the poles of $F$. They appear as the accumulation of the zeros of the polynomials at large enough $N$.
If $R_{F}>R_{K}$, we simply reverse the roles of the series and reconstruct $K(U)$.

This technique has a quite large degree of generality but also limitations. It assumes, for example, that the leading singularities in $F$ are poles and that the radius of convergence of $F$ and $K$ are different. Also, it does not give us indications of poles that would be far from the origin but close to the real axis. However, in practice, we see below that, for the quantities and the physical problem considered in this paper (Green's function and self-energy in real frequency and Kondo temperature), this technique is sufficient. Finally, once $F(U)$ has been resummed, it can be used to locate its zeros and, hence, for the resummation of $K(U)$, which provides another consistency check of the method.

\section{Controlling the noise amplification using nonperturbative information and Bayesian inference}

The transformation from $F_{n}$ to $\bar{F}_{p}$ is a linear one (with a lower triangular matrix), for a given transformation $W(U)$. Depending on the eigenvalues of the corresponding matrix, the Monte Carlo error bar in $F_{n}$ may be strongly amplified by the transformation. As a result, the method may become unusable at strong coupling, as illustrated below in Fig. 6 .

However, if we add some nonperturbative information, such as the fact that the Kondo temperature vanishes at infinite $U$, or a sum rule, we can construct a Bayesian inference technique that may be used to decrease the statistical uncertainty. Bayesian inference provides a systematic and rigorous way to incorporate this information into the results and improve their accuracy. In the rest of this subsection, we describe the general theory for this technique. We illustrate it in the following section.

Let us consider a series $F(U)=\sum_{n=0}^{N} F_{n} U^{n}$, where the $F_{n}$ are known with a finite precision. We denote $F=$ $\left\{F_{0}, F_{1}, \ldots F_{N}\right\}$ the corresponding (vectorial) random variable. We calculate the mean values $\left\langle F_{n}\right\rangle$ and the corresponding errors $\delta_{n}$ within the quantum Monte Carlo technique. We assume that the coefficients $F_{n}$ are given by independent Gaussian variables. The assumption constitutes the "prior" distribution $P_{\text {prior }}[F=f]$ in the absence of additional information:

$$
P_{\text {prior }}[F=f]=\prod_{i=0}^{N} \frac{1}{\sqrt{2 \pi \delta_{n}}} e^{-\frac{\left(f_{n}-\left\langle F_{n}\right\rangle\right)^{2}}{2 \delta_{n}^{2}}} .
$$

Let us note the additional information $X . X$ is a random variable that can be directly calculated from the series, $X=g(F)$, but whose actual value is also known very precisely by other means. In the example below, $X$ is the value of $F(U)$ at large $U$. Bayesian inference amounts to replacing the prior distribution with the posterior distribution $P\left(F=f \mid X=x_{0}\right)$ that incorporates the knowledge of 
the actual value of $X$ [we denote $P(A \mid B)$ the conditional probability of event $A$ knowing event $B$ ]. The value of $X$ is often known exactly. However, due to the presence of truncation errors, its value cannot be enforced exactly, and we suppose that it is known with a small error $\varepsilon$. Eventually, we take the limit $\varepsilon \rightarrow 0$. Hence, we assign to $X$ a Gaussian probability distribution $P_{X}[X=x]=1 /(\varepsilon \sqrt{2 \pi}) \exp [-(x-$ $\left.\left.x_{0}\right)^{2} /\left(2 \varepsilon^{2}\right)\right]$ and define the posterior distribution as

$P_{\text {posterior }}(F=f) \equiv \int d x P(F=f \mid X=x) P_{X}[X=x]$.

Using Bayes formula $P(F=f \mid X=x)=P(X=x \mid F=$ f) $P_{\text {prior }}[F=f] / P_{\text {prior }}[X=x]$ and the deterministic relation $P(X=x \mid F=f)=\delta[x-g(f)]$, one arrives at

$$
P_{\text {posterior }}(F=f)=\frac{P_{X}[X=g(f)] P_{\text {prior }}[F=f]}{P_{\text {prior }}[X=g(f)]} .
$$

In practice, one proceeds as follows: (i) One generates many series according to $P_{\text {prior }}[F=f]$. We emphasize that these series result from a single $\mathrm{QMC}$ run and, hence, are trivially generated (independent Gaussian numbers). Bayesian inference implies no significant computational overhead. (ii) One constructs a histogram of the values of $X$ to obtain $P_{\text {prior }}[X=g(f)]$. (iii) Each series is given a weight $P_{X}[X=g(f)] / P_{\text {prior }}[X=g(f)]$, which is used to calculate other observables such as the value of $F(U)$ at different values of $U$. In practice, the results are insensitive to the choice of $\varepsilon$ as long as it is chosen large enough so that a finite fraction of the sample contributes to the final statistics.

\section{B. Illustration with the Kondo temperature}

Let us first apply the method described above to the Kondo temperature $T_{K}$ (which is $F$ in this section). $T_{K}$ corresponds roughly to the width of the low-energy Kondo peak and is defined more specifically in this paper as the dimensionful Fermi-liquid quasiparticle weight extracted from the retarded self-energy at a low energy:

$$
T_{K}(U) \equiv \frac{2 \Gamma}{1-\left.\partial_{\omega} \operatorname{Re} \Sigma^{R}(U, \omega)\right|_{\omega=0}} .
$$

Our first goal is to illustrate how the method actually works and benchmark it against the calculation of the same quantity from the NRG technique and Bethe ansatz [78,79].

\section{Singularities in the complex $U$ plane}

The dashed blue lines in Fig. 3 show the truncated series of $T_{K}=\sum_{n=0}^{N} F_{n} U^{n}$ for various orders $N \leq 10$. These truncated series diverge around $R_{T_{K}} \approx 5 \Gamma$, which is the convergence radius of the series for these parameters. Increasing the value of $N$ helps to obtain a reliable value of $T_{K}$ closer to $R_{T_{K}}$. However, as expected, even with a very

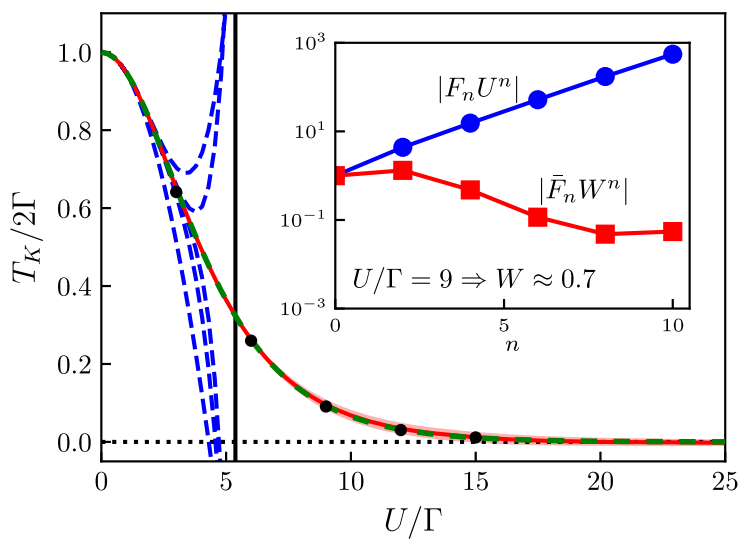

FIG. 3. Resummation of the Kondo temperature [as defined in Eq. (15)] in the symmetric model $\left(\epsilon_{d}=0\right)$. Plain red line: Resummation technique including Bayesian inference, using the Euler transform (error bar shown as a red shaded area); dashed thick green line: exact result from the Bethe ansatz [78]; black circles: reference NRG results; dashed blue lines: truncated series including up to $N=2,4,6,8$, and 10 terms. The vertical line shows the estimated convergence radius of the series. Inset: Evolution of $F_{n} U^{n}$ with $n$ for $U=9 \Gamma$ in the log-linear scale (blue circles); evolution of the series $\bar{F}_{n} W^{n}$ obtained after conformal transformation (red squares). The value $W=0.7$ is obtained by applying the conformal transformation to $U=9 \Gamma$. The $\bar{F}_{n} W^{n}$ decreases exponentially, indicating a convergence of the transformed series while the original series (blue circles) diverges.

large number of terms, the bare series cannot be summed near or above $R_{T_{K}}$. Anticipating the final results, the plain red line corresponds to the results after resummation, which matches very well with what is obtained with our benchmark NRG calculation (see Sec. IV A for details on the used NRG implementation).

The inset in Fig. 3 shows the value of $\left|F_{n} U^{n}\right|$ (blue circles) as a function of $n$ for $U / \Gamma=9$ which lies above the convergence radius of the series. The log-linear plot shows an exponential increase of $\left|F_{n} U^{n}\right| \sim\left(U / R_{T_{K}}\right)^{n}$ with $n$ which we use to extract the convergence radius of the series. Note that, for other series, it can happen that $\left|F_{n}\right|$ oscillates with $n$. Whenever $F_{n}$ changes sign, it becomes close to zero, which provides deviations from the clear exponential behavior shown in the inset in Fig. 3. Hence, to obtain convergence radii which are robust to these outliers, we use a robust regression method on the $\log \left|F_{n}\right|$ versus $n$ data (we compute the regression slope as the median of all slopes between pairs of data points, which is known in statistics as the Theil-Sen estimator [80]).

We now compute the first ten terms of the series of $1 / T_{K}(U)$. This series has a radius of convergence of the order of $10 \Gamma$. We look for the zeros, in the complex plane, of the series $1 / T_{K}(U)$ truncated at the $N$ th order. Since the truncated series is a polynomial, it has (generically) $N$ zeros, which are shown in Fig. 4 for $N=6$ (red squares), $N=8$ (blue circles), and $N=10$ (stars). One pair of zeros 


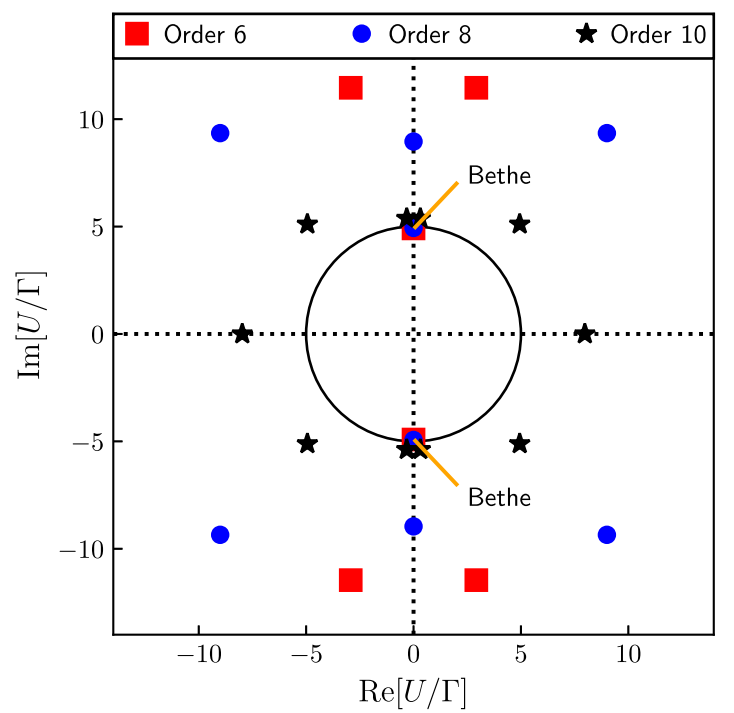

FIG. 4. Poles of $T_{K}(U)$ identified from the zeros of the $1 / T_{K}(U)$ function. These are found by looking for the zeros of its truncated series. Here, they are shown in the $U / \Gamma$ complex plane with truncation at the order of 6 (red squares), 8 (blue points), and 10 (black stars). The black circle corresponds to $|U|=R_{T_{K}}$, where $R_{T_{K}}$ is the radius of convergence of the series of $T_{K}$. The stable points close to $\pm i 5 \Gamma$ correspond to true nonperturbative poles of $T_{K}(U)$. The exact zeros (small orange arrows) are computed from the exact $1 / T_{K}$ series found with the Bethe ansatz [78].

$U \approx \pm i 5 \Gamma$ is converged for all the truncations and, hence, corresponds to a true zero of $1 / T_{K}(U)$, i.e., to a pole of $T_{K}(U)$. Figure 4 also shows the circle $|U|=R_{T_{K}}$ extracted from the analysis of the $T_{K}(U)$ series done in the inset in Fig. 3. We find that the two poles $\pm i 5 \Gamma$ do indeed lie right on this circle.

\section{Conformal transformation}

Let us now turn to the conformal transformation $W(U)$, which maps the two poles $\pm i 5 \Gamma$ away and brings the values of interest $U>0$ (real) closer to zero. We illustrate the technique with two maps: the Euler map defined by

$$
W=\frac{U}{U-p}
$$

and the "parabola" map, which is defined as

$$
W=-\tan ^{2}\left(\frac{\pi}{2} \sqrt{\frac{U}{p}}\right),
$$

where $p$ is an adjustable complex parameter.

Figure 5 shows the various regions (different colors) in the $U$ plane that are mapped onto concentric circles of the $W$ plane. 0 is mapped onto 0 and $p$ onto $\infty$ in both transforms. The Euler map (left column) maps one half of
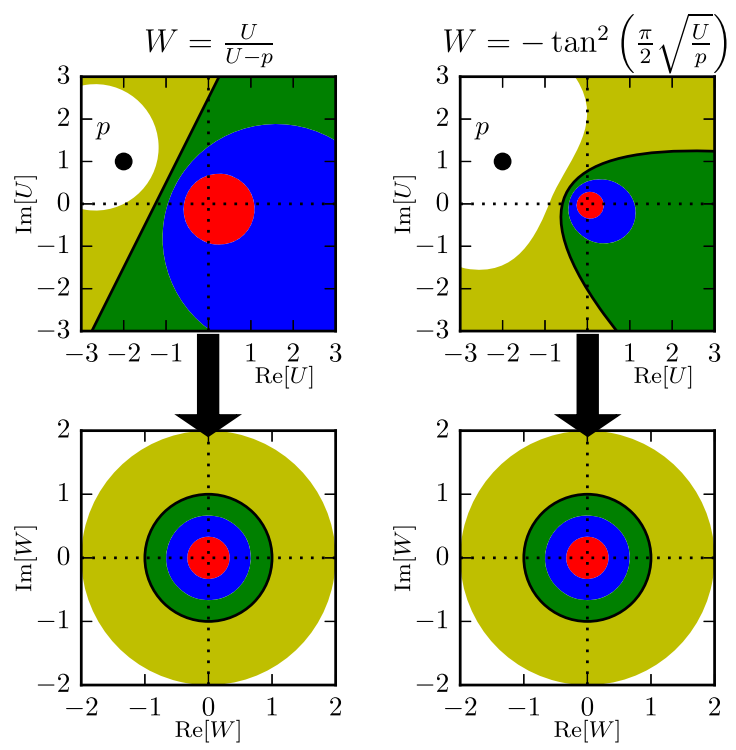

FIG. 5. Left: Euler map. Right: Parabola map. Top: Complex $U$ plane. Bottom: Complex $W$ plane. The transformation maps the top regions of various colors onto the bottom regions of matching colors. In particular, the thick straight line (top left) and the parabola (top right) are mapped onto the unit circles (bottom left and right, respectively).

the plane into the unit disk and the other half into the outside of the unit disk (separated by a black line). The parabola transform (right column) maps the inside of a parabola (black line) into the unit disk and the outside of the parabola into the outside of the unit disk. In the case where there are no singularities on the positive half plane $\operatorname{Re}[U]>0$, the Euler transform should be preferred, since real values of $U>0$ are typically mapped closer to $U=0$ than with the parabola transform (compare the size of the blue region of the parabola and Euler case, for instance). However, the parabola map is more agnostic about the positions of the singularities and will work even if there are singularities on the positive half plane $\operatorname{Re}[U]>0$ as long as they lie outside the parabola.

We now perform the resummation of $T_{K}(U)$. The series contains only even power of $U$ due to particle-hole symmetry, so that it can be considered as a function of $U^{2}$. The two poles $U= \pm i 5 \Gamma$ correspond to a single one $U^{2}=-25 \Gamma^{2}$. In the $U^{2}$ plane, the pole being on the negative real axis, the Euler maps works very effectively. The resummation can also be performed with the parabola transform.

Once the conformal map is selected, we form the series $\bar{F}_{p}$ in the $W$ variable, as explained above. The inset in Fig. 3 shows $\bar{F}_{n} W_{0}^{n}$ (red squares) as a function of $n$ for $W_{0}=0.7=W\left(U_{0}=9 \Gamma\right)$, using the Euler map with $p=$ $-35 \Gamma^{2}$ (the parabola yields similar results with $p=-15 \Gamma^{2}$ ). As expected, $U_{0}$ is way beyond the radius of convergence in the original variable $U$, while $W_{0}$ lies within the disk of convergence of $\bar{F}(W)$ whose radius is found to be $R_{\bar{F}} \approx 2$. 
The final result $T_{K}(U)$ using the Euler transforms is shown in Fig. 3. The parabola transform (not shown) is undistinguishable from the Euler at this scale.

In this work, singularities are never found near the real positive axis, so that all $U>0$ can be reached using the conformal transforms in Fig. 5, given that enough orders of the series are known. However, one may very well build a conformal transform to reach a regime beyond a singularity by considering a concave contour $\mathcal{C}$, as shown in the Appendix A. This approach may become interesting if a phase transition occurs when interaction is increased.

\section{Noise reduction with Bayesian inference}

Let us now apply the Bayesian inference technique described above to the computation of $T_{K}(U)$. In the left in Fig. 6, we resample the series for the Kondo temperature; i.e., we generate many series (typically, $10^{3}-10^{5}$ samples). For each sample, we perform the conformal transformation and plot the result for the Kondo temperature as a function of $U$ (thin red lines). While we find that all results agree for $U \leq 6 \Gamma$, the bundle of curves starts to diverge for larger values of $U$. In the middle, we plot (black thin line) the corresponding histogram of the values obtained for $T_{K}(U=\infty)$, which is $P_{\text {prior }}\left[T_{K}=g(f)\right]$.

We use the nonperturbative relation $\lim _{U \rightarrow \infty} T_{K}(U)=0$. Hence, we want to "postselect" the configuration of $F_{n}$ which gives a vanishing Kondo temperature at large $U$, at precision $\varepsilon$. Following the procedure described in Sec. III A 3, our final result is obtained by averaging the different traces (thin red lines) with the weight given by Eq. (14). The right in Fig. 6 shows the result for three different values of $U$ as a function of $\varepsilon$, which confirms that the results are insensitive to the actual value of $\varepsilon$. We find a very good agreement with the results obtained from the NRG even at large values of $U$, noting that NRG spectra have typical relative error bars of a few percents (see Sec. IVA for details).

\section{Benchmark with the Bethe ansatz exact solution}

The series expansion for $1 / T_{K}(U)$ is calculated explicitly and exactly using the Bethe ansatz technique by Horvatić and Zlatić [78,79]. Reference [78] provides an iterative formula for calculating the coefficients of the expansions and shows that the corresponding series has an infinite radius of convergence. This result provides another independent benchmark of the calculation of $T_{K}(U)$ as well as of the method itself. We check that the ten first coefficients of this series agree with the one that we compute with QMC.

Figure 3 shows our final result together with the NRG result (black circles) and the Bethe ansatz results. At this scale, the agreement is perfect. Using the exact series for $1 / T_{K}(U)$ (truncated to around 50 coefficients), we study its zeros, which are the poles of $T_{K}(U)$. We find that they are situated on the imaginary axis. The poles closest to the
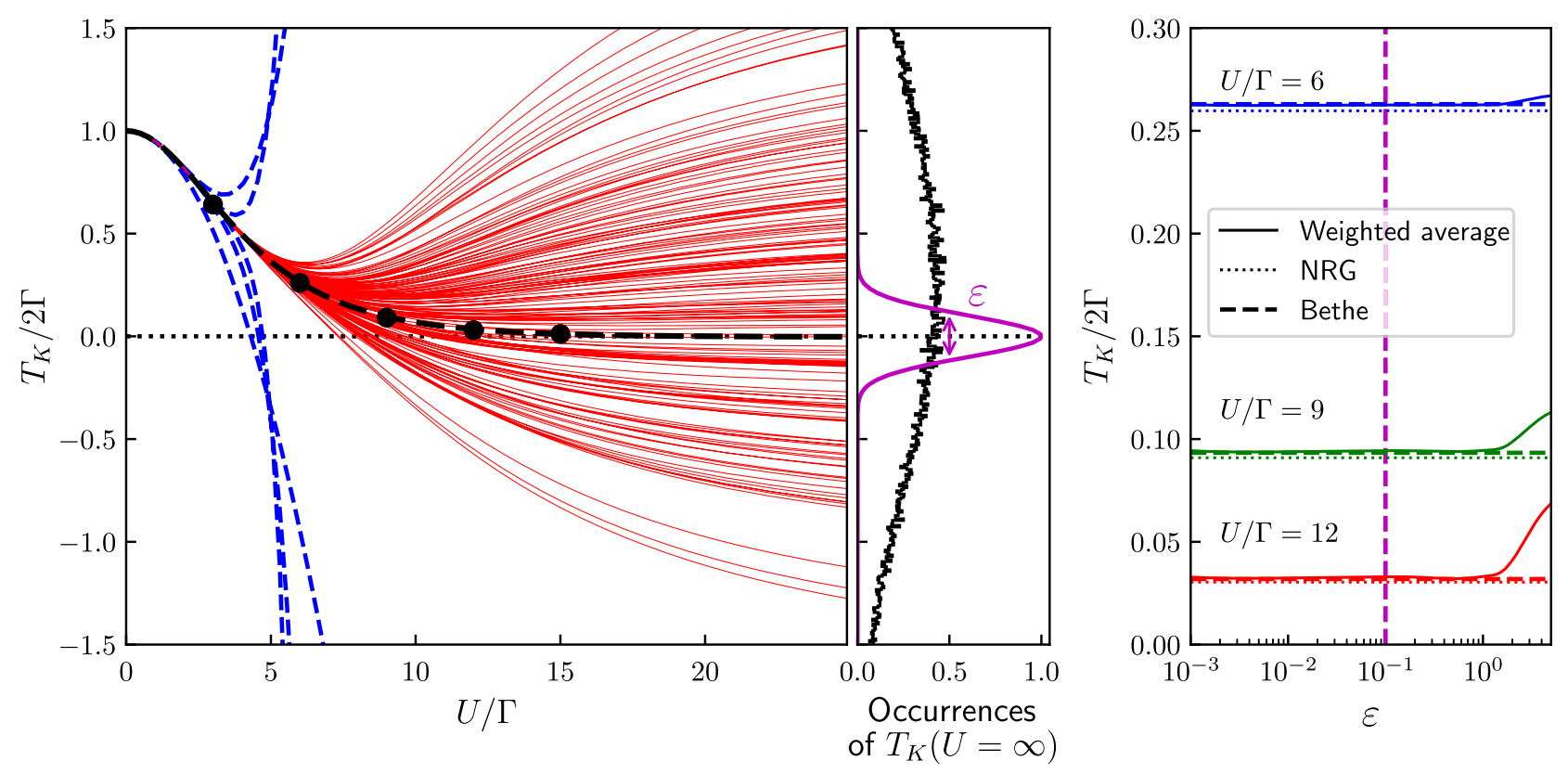

FIG. 6. Reduction of the statistical noise on the resummed $T_{K}(U)$ series by Bayesian inference. Left: Kondo temperature as a function of $U$. The bundle of red lines corresponds to different samples of our series after resummation (see the text). The thick line shows the final result after Bayesian inference, while the circles show our reference NRG calculations. The dashed blue lines show the bare results without resummation, which diverge for $U>5 \Gamma$. Middle: Histogram of the values of $T_{K}(U=\infty)$ obtained from our samples (black line) and a histogram of its assumed distribution with tolerance $\varepsilon$ (purple line). Right: The final result after inference as a function of $\varepsilon$ for three values of $U / \Gamma=6,9$, and 12 (thin lines), the reference NRG result (dotted lines), and the Bethe ansatz result (dashed horizontal lines). 
origin are $U / \Gamma \approx \pm 4.89059579 i$ in agreement with our findings; see Fig. 4 . The next poles are $U / \Gamma \approx \pm 13.79 i$, $21.77 i, 29.89 i, 37.87 i$, and $45.9 i$ but are too far to be accessible with only the first ten coefficients. The right in Fig. 6 provides a detailed benchmark of our results versus both the NRG and the exact Bethe ansatz solution.

We find that the QMC results for $T_{K}$ are slightly more accurate than the NRG, because the extraction of $T_{K}$ from the NRG self-energy [see Eq. (15)] contains inherent broadening errors. The agreement between all three methods is nevertheless excellent. In addition, we can extract from the Bethe ansatz the exact QMC error, and this error matches the measured one-sigma statistical error bars.

\section{Equilibrium dynamical correlation functions}

Let us now apply our method to the Green's function and self-energy as a function of the real frequency $\omega$.

\section{Singularities in the long time (stationary) limit}

Let us now turn to the full Green's function $G^{R}(\omega, U)$ and self-energy $\Sigma^{R}(\omega, U)$. An example of our bare data is shown in Fig. 2, where we plot the coefficients $\Sigma_{n}^{R}(\omega)$ obtained from the real time diagrammatic quantum Monte Carlo algorithm for $n=2,4,6,8$, and 10. The description of the method used to calculate these coefficients $\Sigma_{n}^{R}(\omega)$ is explained in the companion paper to this article [64].

We focus on the quantity $\Sigma^{R}(\omega)-i \Gamma$ and denote its inverse $F_{\omega}(U)=1 /\left[\Sigma^{R}(\omega)-i \Gamma\right]$. The retarded Green's function can be recovered from $F_{\omega}(U)$ using $G^{R}(\omega)=$ $1 /\left[\omega-F_{\omega}(U)^{-1}\right]$ [using $\omega-\Sigma^{R}(\omega)+i \Gamma$ turns out to be less convenient especially at high frequencies].

Figure 7 shows the convergence radius of $F_{\omega}(U)$ as a function of the frequency, extracted from a study of the exponential decay of the corresponding series with $n$. We also perform a systematic study of the zeros of $\Sigma^{R}(\omega)-i \Gamma$ in order to localize the poles of $F_{\omega}(U)$. We find one pair of poles at each frequency. The results are shown in the inset in Fig. 7 for a set of frequencies from $\omega=0$ to $\omega=10 \Gamma$ in the complex plane for $U^{2}$. The absolute value of the poles of $F_{\omega}(U)$ is also plotted in the main frame in Fig. 7 as a function of the frequency (circles of varying colors from blue to red). We observe a perfect match with our estimation of the convergence radius, reflecting the fact that these poles are responsible for the divergence of the series. It is important to note here that working in the real frequency domain is very helpful: We find a single pole per frequency (at least for the range of interactions that we could study). Hence, we expect that performing the resummation in real time or imaginary frequencies could be more complex, since all these poles would be involved simultaneously.

The results for three frequencies $(\omega / \Gamma=1,2$, and 6$)$ are given in Fig. 8. We show the convergence of the imaginary part of the self-energy using two different resummed series:

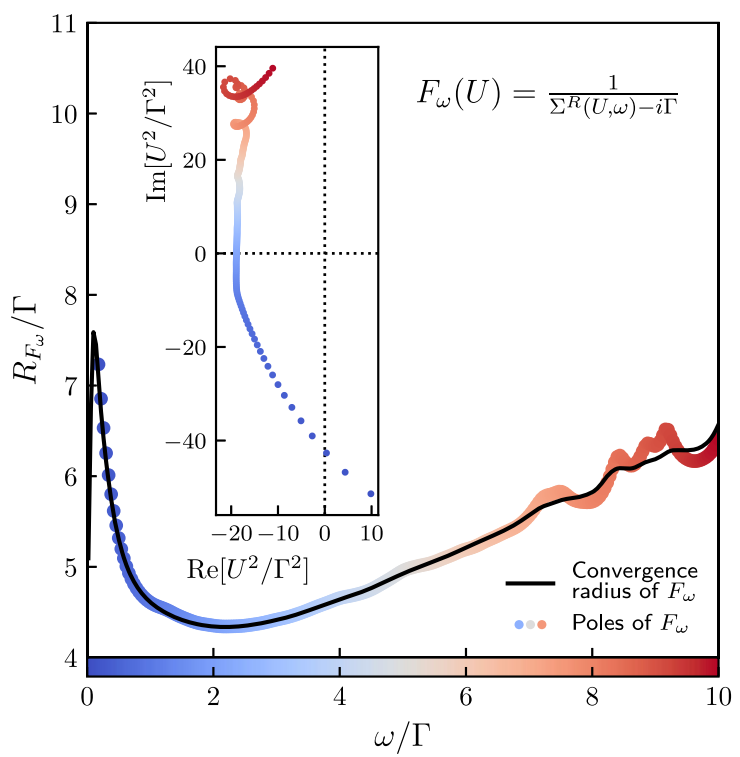

FIG. 7. Main frame: Convergence radius $R_{F_{\omega}}$ of $F_{\omega}(U)=$ $1 /\left[\Sigma^{R}(U, \omega)-i \Gamma\right]$ (thin line) in the equilibrium symmetric Anderson impurity model. The color circles show the absolute value of the pole of $F_{\omega}(U)$. Inset: Position of the pole of $F_{\omega}(U)$ in the $U^{2}$ complex plane for different frequencies. The color blue to red corresponds to the increasing frequency, as in the main frame. At high frequencies, the statistical uncertainty prevents an accurate localization of the poles.

$F_{\omega}(U)$ (green symbols) and $1 / F_{\omega}(U)$ (purple symbols). The former is resummed with an Euler transform with a frequency-dependent $p$ set close to the poles shown in Fig. 7. The latter, for which our method does not detect poles, is resummed with the parabola transform (in the $U$ plane) with $p=-4.5 \Gamma$. Again, Bayesian inference is used to enforce $\lim _{U \rightarrow \infty} G(U, \omega)=0$ for all $\omega \neq 0$.

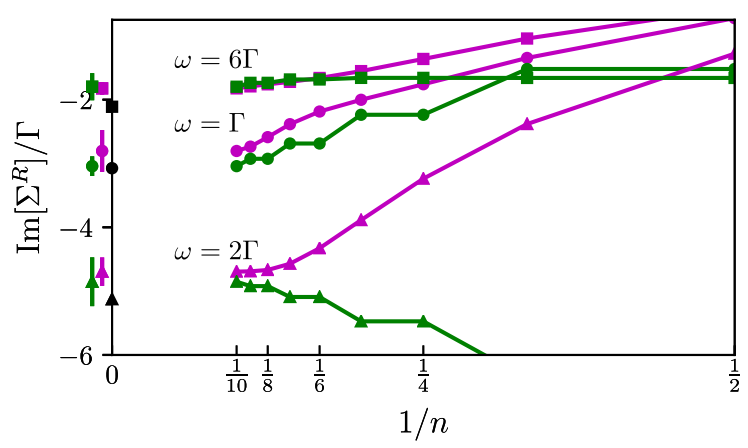

FIG. 8. Resummation of the self-energy in the equilibrium symmetric Anderson impurity model at $U=9 \Gamma$. The imaginary part of $\Sigma^{R}(\omega)$ is shown as a function of the number $n$ of terms kept in the resummation, for three frequencies $\omega=\Gamma$ (circles), $2 \Gamma$ (triangles), and $6 \Gamma$ (squares). The independent resummations of $F_{\omega}(U)$ (green line) and of $\Sigma^{R}(\omega)-i \Gamma$ (purple line) converge with one another. The results with truncation and statistical errors are shown on the left of the $y$ axis, along with NRG results (black symbols). 
For comparison, we also include the NRG results (which are very accurate at small frequencies and possibly less accurate at large frequencies). The slight difference between the purple and green curves is due to the truncation error. We find that the series which has (initially) the largest convergence radius is less sensitive to truncation error or statistical noise than the other. We attribute the small discrepancy between the QMC results and NRG at large frequencies to a lack of convergence of the latter. These results are obtained for a rather strong interaction $U=9 \Gamma$. At smaller interactions, the QMC and NRG results become undistinguishable. At larger interactions, the QMC results become increasingly inaccurate due to truncation errors.

\section{The long time limit}

In the Keldysh formalism, the interactions are switched on at an initial time $(t=0)$, and one follows the evolution of the system with time $t$. We assume here that the system relaxes to a steady state at a long time. Let us consider the average of an operator $\hat{\mathcal{O}}$ as a function of the time, and its expansion $\langle\hat{\mathcal{O}}(t)\rangle=\sum_{n} O_{n}(t) U^{n}$ (the extension of the following arguments to the Green's function is straightforward).

At finite time $t$, the radius of convergence of this series is infinite, as shown in Appendix B. Each order in the perturbation expansion $O_{n}(t)$ relaxes with $t$ to a long time limit, but the time $t_{\text {relax }}[n]$ it takes to reach this limit can increase with $n$. The long time and large $n$ limit do not commute, in general:

$$
\lim _{n \rightarrow \infty} \lim _{t \rightarrow \infty} O_{n}(t) \neq \lim _{t \rightarrow \infty} \lim _{n \rightarrow \infty} O_{n}(t) .
$$

This behavior was already noted in Fig. 14 in Ref. [39]. It is also illustrated in Fig. 9, which shows various orders $n$ of the expansion of the current through the dot versus $n$, for different times. We observe that at small times the orders $I_{n}$ decrease faster than exponentially with $n$, consistent with the bound mentioned above. The coefficients converge to the steady-state limit at long time.

At finite time $t$, since the series converges, it is sufficient to have enough orders. In the steady state, as explained above, we have a minimal order $N_{0}$ needed to compute the quantity at a given precision. One should then simply compute at a time $t>t_{\text {relax }}\left[N_{0}\right]$.

In the Anderson model, some quantities like the spectral function are known to relax on a long timescale $t_{K} \sim T_{K}^{-1}$; see, e.g., Ref. [81]. The previous remarks explain how the algorithm deals with this long time. For a given $U$, we need $N_{0}(U)$ orders, hence, to compute at a time larger than $t_{\text {relax }}\left[N_{0}(U)\right]$. The larger $U$ is, the longer this time becomes. However, it is still finite at fixed $U$, and, since our calculation of the perturbative expansion is uniform in time, it is not an issue (the computation effort does not grow with time). However, the existence of the Kondo time

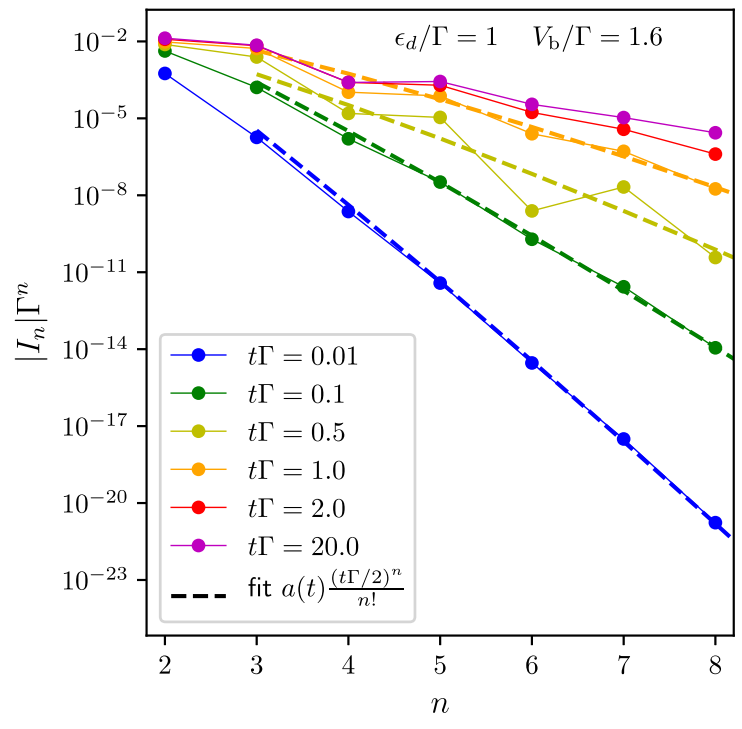

FIG. 9. Coefficients (absolute value) of the series for the current (circles with thin lines) in the asymmetric model $\left(\epsilon_{d}=\Gamma\right.$, $\left.V_{b}=1.6 \Gamma\right)$ computed at different times $t$ (different colors). The apparent convergence radius decreases with time. For small values of $t$, we can observe that the series coefficients decrease faster than exponentially, which indicates an infinite convergence radius. The thick dashed line shows the corresponding fit with $(t \Gamma / 2)^{n} / n$ !. For large enough $t$, the series converges toward the steady-state limit.

indicates that the number of orders necessary to compute, e.g., the low-frequency spectral function at a given $U$ increases with $U$ (otherwise, the relaxation time of the physical quantity would be bounded at large $U$ ).

\section{BENCHMARK OF THE DYNAMICS IN EQUILIBRIUM}

We now benchmark our results in the case of equilibrium, testing various regimes of the Anderson impurity model. Let us first describe the high-precision NRG computations that are performed.

\section{A. NRG implementation}

The NRG [82] is used to benchmark our QMC calculations in equilibrium and to test the reliability of the series extrapolation method for spectral functions at various values of $U$ and $\epsilon_{d}$. In order to obtain precise NRG data for the spectral function of the Anderson impurity model, the computations are performed using several improvements over the simplest implementations of the NRG. First, the full density matrix formulation of the NRG [83] is used to reduce finite size effects due to the NRG truncation. Second, symmetries of the problem are heavily exploited [84], allowing one to reduce significantly the Hilbert space dimension of various multiplets. In the particle-hole symmetric case, the full $\mathrm{SU}(2)_{\text {charge }} \otimes \mathrm{SU}(2)_{\text {spin }}$ symmetry is used, while the charge sector is reduced to $\mathrm{U}(1)_{\text {charge }}$ away 
from particle-hole symmetry. Third, the impurity Green's function is extracted from a direct computation of the $d$-level self-energy $\Sigma(\omega)$ [85], according to its exact representation as the ratio of two retarded correlation functions in the frequency domain:

$$
\Sigma(\omega)=U \frac{F^{R}(\omega)}{G^{R}(\omega)},
$$

where $G^{R}(t)=-i \theta(t)\left\langle\left\{d_{\sigma}(0), d_{\sigma}^{\dagger}(t)\right\}\right\rangle$ is the usual singleparticle retarded Green's function in the time domain and $F^{R}(t)=-i \theta(t)\left\langle\left\{d_{\sigma}(0) d_{-\sigma}^{\dagger}(0) d_{-\sigma}(0), d_{\sigma}^{\dagger}(t)\right\}\right\rangle$ is a composite fermionic correlation function. In practice, $\operatorname{Im}\left[G^{R}(\omega)\right]$ and $\operatorname{Im}\left[F^{R}(\omega)\right]$ are computed from the Källén-Lehmann representation using the broadened NRG spectra, and the real parts of both $G^{R}(\omega)$ and $F^{R}(\omega)$ are obtained via a Kramers-Kronig relation. Finally, the truncation parameters of the NRG simulations are taken to model as closely as possible a continuous density of states for the electronic bath. Although the use of the logarithmic Wilson discretization grid, $\omega_{n}=D \Lambda^{-n}$, is inherent to the practical success of NRG, we find that values of $\Lambda$ as low as $\Lambda=1.4$ can be managed in practice within the NRG, taking a very large number $N_{\text {kept }}=3200$ of kept multiplets. Up to $N_{\text {iter }}=120 \mathrm{NRG}$

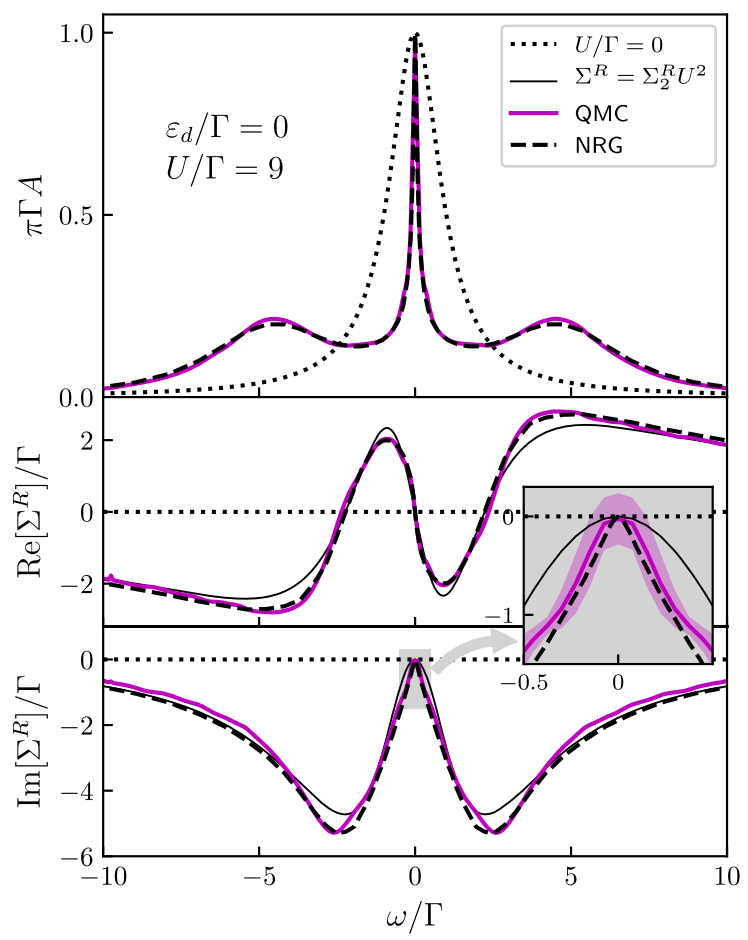

FIG. 10. Resummed equilibrium spectral function (top), real part (middle), and imaginary part (bottom) of the retarded selfenergy $\Sigma^{R}(\omega)$ for the symmetric Anderson impurity $\epsilon_{d} / \Gamma=0$ at $U=9 \Gamma$. Purple line: Resummed result from 10 orders of perturbation theory; dashed line: NRG; dotted line: noninteracting result; thin black line: second-order perturbation theory for the self-energy. Inset: Enlargement of the imaginary part at a small energy with error bars. iterations are used, so that the effective temperature can be considered to be practically zero. With such a small value of $\Lambda$, the broadening parameter $b$ of the discrete NRG spectra could be decreased down to $b=0.2$, without $z$ averaging, which further enhances the spectral resolution of the Hubbard satellites in the spectral function.

\section{B. Comparison to NRG in equilibrium}

Figure 10 shows the spectral function as well as the imaginary and real parts of the self-energy for the symmetric Anderson impurity in the strong correlation regime $U=9 \Gamma$ (the same data as the purple curve in Fig. 8). The spectral function shows a clear Kondo peak and the two satellites at $\omega \simeq \pm 4.5 \Gamma= \pm U / 2$ in good agreement with the NRG data. For this calculation, a simple second-order calculation of the self-energy already provides a reasonably good result (thin black line), due to near cancellations in higher-order diagrams in the peculiar case of particle-hole symmetry.

Figure 11 shows the same plot in the asymmetric case $\epsilon_{d}=1$. This case is more complex, because the resonance at $U=0$ is offset with respect to the Fermi level and, hence, to the position of the Kondo peak. We note that previous real-time $\mathrm{QMC}$ techniques suffer from a strong sign problem and cannot access the asymmetric regime

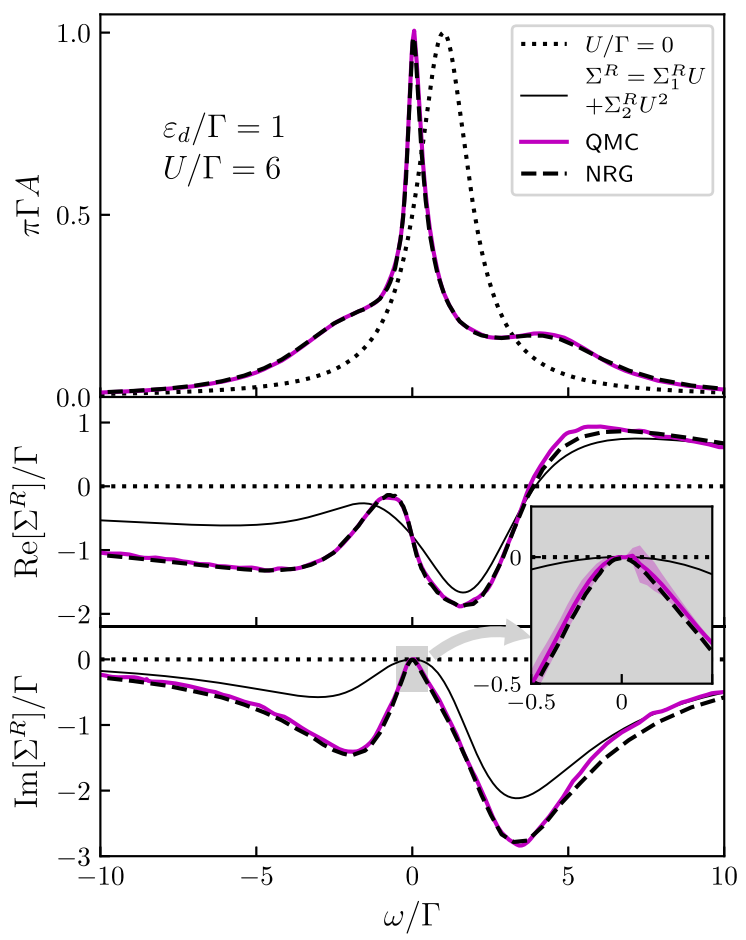

FIG. 11. Resummed equilibrium spectral function (top), real part (middle), and imaginary part (bottom) of the retarded selfenergy $\Sigma^{R}(\omega)$ for the asymmetric Anderson impurity $\epsilon_{d} / \Gamma=1$ at $U=6 \Gamma$. Purple line: Resummed result from 10 orders of perturbation theory; dashed line: NRG; dotted line: noninteracting result; thin black line: second-order perturbation theory for the self-energy. Inset: Enlargement of the imaginary part at a small energy with error bars. 


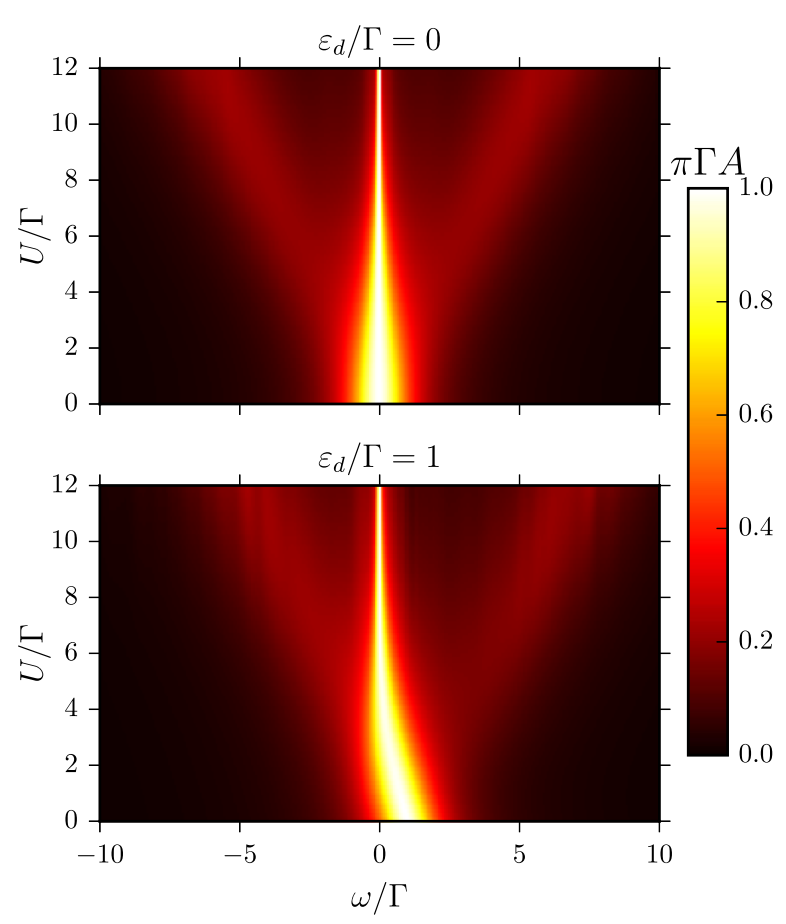

FIG. 12. Color plot of the spectral density $A(\omega, U)$ in the symmetric case $\left(\epsilon_{d} / \Gamma=0\right.$, upper $)$ and asymmetric case $\left(\epsilon_{d} / \Gamma=1\right.$, lower) as a function of $\omega$ and $U$. The data from each panel are obtained in a single QMC run.

[31]. We also stress that the second-order approximation is now very different from the correct result. The comparison to the NRG data is still excellent.

Another advantage of the techniques described in this article and its companion article [64] is that a single QMC run provides the full dependence in both $\omega$ and $U$, which is very time consuming in the NRG. Hence we show in Fig. 12 the spectral function as a function of $\omega$ and $U$. One can clearly observe the formation of the Kondo peak (which gets thinner as one increases $U$ and shifts toward $\omega=0$ in the asymmetric case) as well as the Hubbard bands at $\omega= \pm U / 2$. Note that the results are perfectly well behaved (qualitatively correct) up to very large $U$ (even above $U=$ $12 \Gamma$ shown in the plot) but become quantitatively inaccurate at too large values of $U$. Improving them would require the use of higher perturbation orders.

The bare power series for the Green's functions computed with QMC in this section can be found in Supplemental Material [86].

\section{OUT-OF-EQUILIBRIUM RESULTS}

We finally turn to the out-of-equilibrium regime and present some accurate computation of current-voltage characteristics, as well as novel predictions for dynamical observables in the presence of a finite bias voltage.

The bare power series for the nonequilibrium Green's functions computed with QMC for typical sets of parameters can be found in Supplemental Material [86].

\section{A. Splitting of the spectral function}

Figure 13 shows the spectral function of the symmetric impurity in the presence of various bias voltages from $V_{b}=$ 0 to $4 \Gamma$. The results are obtained using the parabolic map on the series of $\Sigma_{\omega}\left(U^{2}\right)-i \Gamma$ (with an optimized frequencydependent parameter $\left.p / \Gamma^{2} \in[-25,-200]\right)$. Upon increasing the bias voltage, we find as expected from the NCA [22] and perturbative [20] calculations that the Kondo resonance simultaneously broadens and gets split into two peaks. Previous results on the spectral function [35] are based on the bold diagrammatic approach and are calculated at a relatively high temperature $(T=\Gamma / 3)$ while using a third terminal for computing the spectral function.

Most of the results of this paper are obtained at a very low temperature. We emphasize, however, that increasing the temperature makes the calculations easier: Indeed, at a finite temperature, the noninteracting Green's functions decrease exponentially as $e^{-t / \beta}$ instead of the algebraic decay at a zero temperature. It follows that the support of the integrals to be calculated is smaller and, hence, the convergence of the calculation faster. We show a calculation at a finite temperature in Fig. 14, where we compute the spectral density of the symmetric impurity at temperature $T=\Gamma / 50$ under a bias voltage $V_{b}=0.6 \Gamma$ and $V_{b}=1.5 \Gamma$. A single Monte Carlo run allows us to observe the splitting of the Kondo resonance as $U$ is increased (top). The result is quantitatively accurate up to $U \approx 8 \Gamma$ (bottom) but remains qualitatively meaningful at a higher interaction (top).

The fate of the Kondo resonance out of equilibrium, in the presence of a bias voltage, can be understood

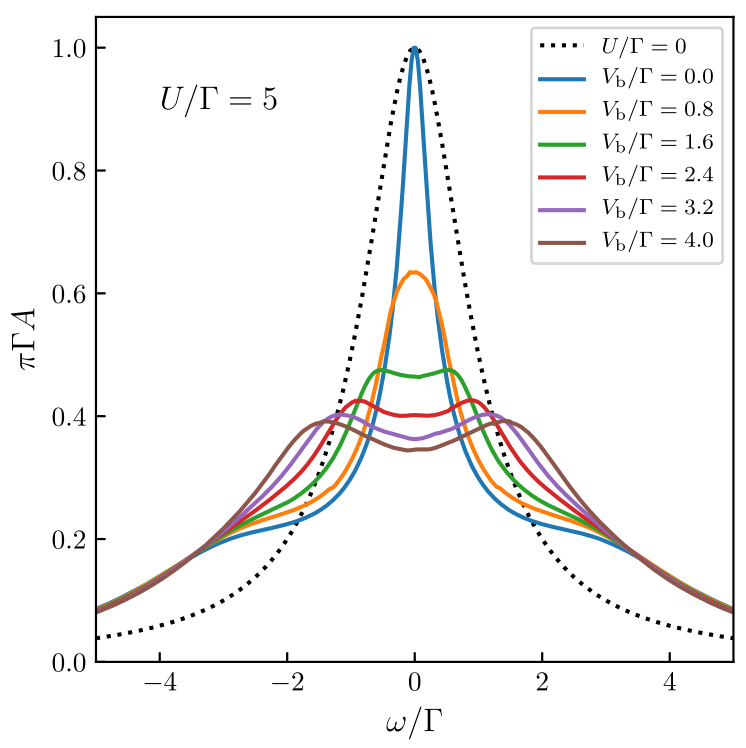

FIG. 13. Out-of-equilibrium spectral functions with interaction strength $U / \Gamma=5$, in the symmetric $\left(\epsilon_{d} / \Gamma=0\right)$ model with a symmetric voltage bias $V_{b}$. The resulting self-energy series is resummed in a similar fashion as for the previous results. The noninteracting spectral function is shown as a dotted line. 

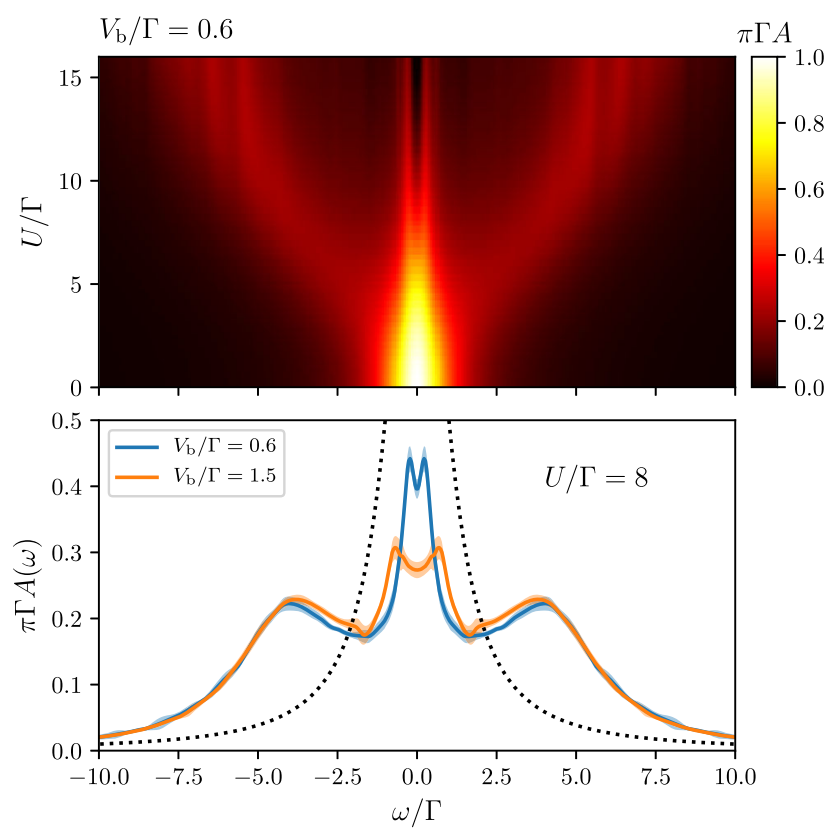

FIG. 14. Out-of-equilibrium spectral functions of the impurity with the same parameters as in Fig. 13, except for temperature $T=\Gamma / 50$. Top: Color plot of the spectral density as a function of $\omega$ and $U$ for a voltage bias $V_{b}=0.6 \Gamma$. Bottom: Spectral density at $U=8 \Gamma$ for a bias $V_{b}=0.6 \Gamma$ (blue line) and $V_{b}=1.5 \Gamma$ (orange line). Error bars are shown as shaded areas. The dotted line shows the noninteracting density. No Bayesian inference is used. The integration time is $20 / \Gamma$.

qualitatively from the interplay of two phenomena. On the one hand, the bias voltage induces a splitting of the Fermi energies of the two reservoirs; hence, one expects a corresponding splitting of the Kondo resonance. On the other hand, the voltage, like the temperature, increases the energy and phase space for the spin fluctuations, leading eventually to the disappearance of the Kondo resonance [87-89]. The competition between both effects leads to the appearance of the splitting only above a finite voltage threshold (about $V_{b} \simeq \Gamma$ in the plot in Fig. 13).

\section{B. $I-V$ transport characteristics}

Figure 15 shows the results obtained for the $I-V$ characteristics in the symmetric case $\epsilon_{d}=0$. The resummation is done for the series of $1 / I\left(U^{2}\right)$ using a parabolic transform with $p=-40 \Gamma^{2}$. At small bias, we recover a perfect transmission $I=\left(e^{2} / h\right) V_{b}$ due to the unitary Kondo resonance, while for $e V_{b}>k_{B} T_{K}$ the conductance experiences an extra suppression by the interaction (Coulomb blockade). We find a very good match with a previous calculation from Ref. [31]. The present technique allows one to lift the main limitations that Refs. [30,31] face: We can now access long times (here, we use $\sim 20 / \Gamma$, but it could be increased further if necessary) to be compared with maximum times of the order of $\sim 3-5 / \Gamma$ in Ref. [31]. As a consequence, we can reach the low bias

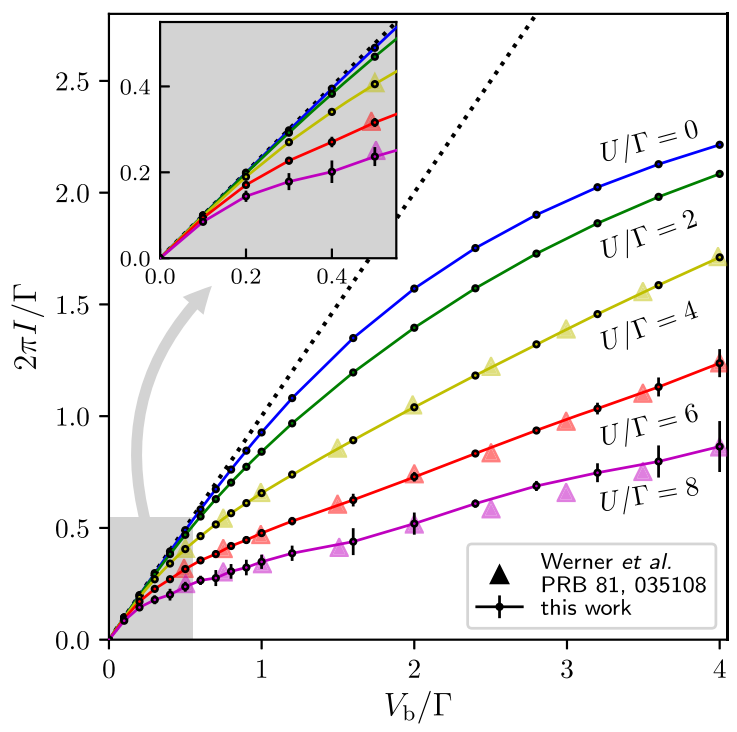

FIG. 15. Current-voltage characteristics at different interaction strengths in the symmetric case $\epsilon_{d}=0$. Perturbation series for the current are computed using the Landauer formula Eq. (7) and then resummed. The results are consistent with a weak-coupling quantum Monte Carlo calculation from Werner et al. [31] (triangles) but extends further down in bias.

regime, which is not accessible in Ref. [31]. Another important point is that the method is not limited to the symmetry point, as we now demonstrate.

Figure 16 shows the $I-V$ characteristics for an asymmetric model with $\epsilon_{d} / \Gamma=1$. The results are obtained from the resummation of $1 / I(U)$ with a parabolic transform $(p=-6 \Gamma)$ and no Bayesian inference. The $I-V$

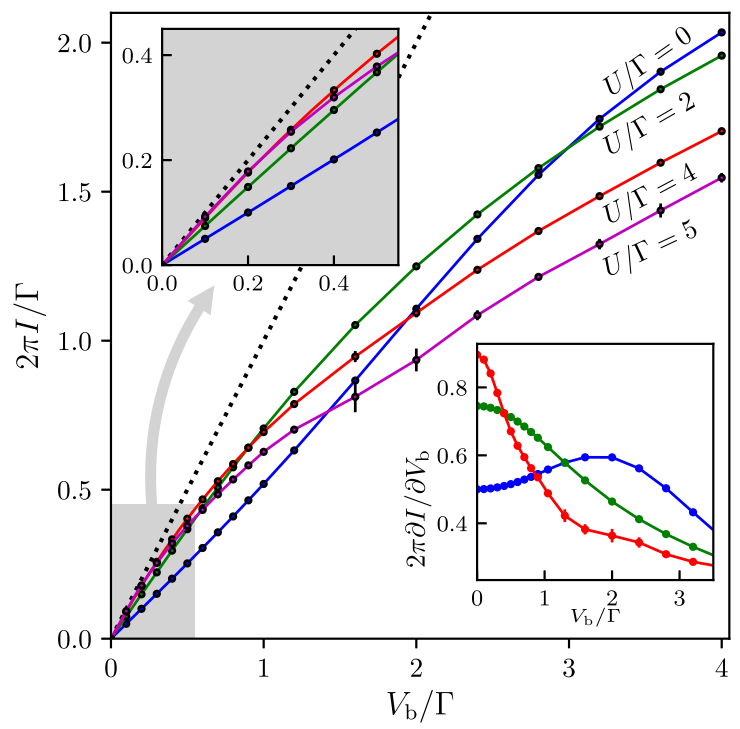

FIG. 16. Current-voltage characteristics at different interaction strengths in the asymmetric model $\left(\epsilon_{d} / \Gamma=1\right)$. The bottom-right inset shows the development of the zero-bias anomaly in the differential conductance when $U$ increases $(U / \Gamma=0$ in blue, 2 in green, and 4 in red). 

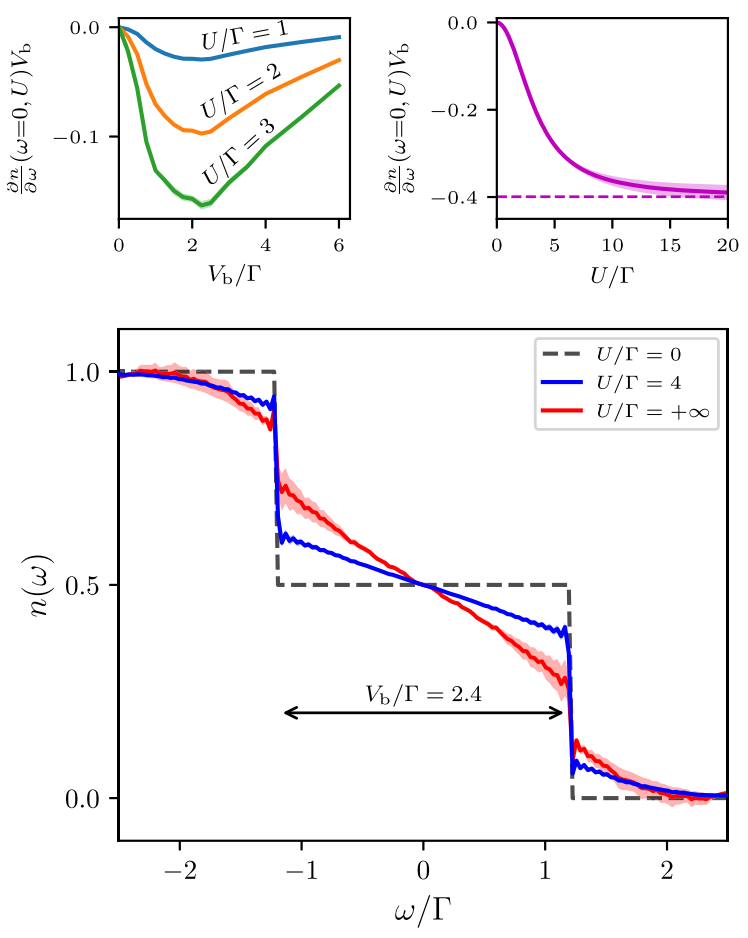

FIG. 17. Bottom: Out-of-equilibrium electron distribution function on the impurity $\left(\epsilon_{d} / \Gamma=0\right)$ under a bias voltage $V_{b}=2.4 \Gamma$. The distribution function is defined as in Eq. (20). Increasing the interaction strength $(U / \Gamma=4$ blue line, $U / \Gamma=+\infty$ red line) leads to a softening of the characteristic double step of the noninteracting distribution function (dashed line). It is linear between the Fermi levels of the two leads. The Euler transform is used for resummation, and the result is not submitted to Bayesian inference. Top: Normalized slope of the distribution function near $\omega=0$ as a function of the bias voltage (left) and interaction strength (right). For an intermediate interaction, the normalized slope reaches an extremum near $V_{b}=2 \Gamma$ (left). At a strong interaction, the normalized slope saturates (right, for $V_{b}=2.4 \Gamma$ ).

characteristics is particularly interesting, because, due to the asymmetry, the noninteracting low bias transmission is modified by interactions, and one must first build up the Kondo resonance to approach $I \simeq\left(e^{2} / h\right) V_{b}$ (note that the unitary limit is strictly exact only at $\epsilon_{d}=0$, and the conductance is slightly lower than $e^{2} / h$ otherwise in the Kondo regime). This behavior leads to a nonmonotonic current versus $U$ : As one increases $U$, the current first increases until the Kondo resonance is fully built (see the bottom in Fig. 12). As one increases further $U$, the Kondo width $T_{K}$ shrinks and the current decreases as Coulomb blockade starts to set in.

\section{Biased distribution function}

Finally, we discuss the out-of-equilibrium distribution function of the impurity, i.e., its energy-dependent probability of occupation. We define the distribution function $n(\omega)$ as

$$
n(\omega)=\frac{G^{<}(\omega)}{2 \pi i A(\omega)},
$$

so that at equilibrium $n(\omega)$ is simply the Fermi function $n_{F}(\omega)$. Without an interaction, the distribution function amounts (at a zero temperature) to a double-step function $n(\omega)_{U=0}=\left[n_{F}\left(\omega-V_{b} / 2\right)+n_{F}\left(\omega+V_{b} / 2\right)\right] / 2$. We want to investigate the behavior of $n(\omega)$ as $U$ increases, a question that is not addressed in the previous literature to the best of our knowledge.

The results are shown in Fig. 17. In this particular case, the series are fully alternated, which means that the singularity lies on the negative real axis. We could sum the series using an Euler transform ( $p=-8 \Gamma^{2}$ ) up to $U=+\infty$. We find that the function $n(\omega)$ is not thermal; i.e., it cannot be fitted by a Fermi function $n_{F}$ with an effective temperature. In particular, it still exhibits discontinuities at the position of the lead Fermi surfaces, which we expect to be rounded at a finite temperature. Interestingly, these discontinuities are comparable to the equilibrium quasiparticle weight for $U=4 \Gamma$ and do not seem to vanish in the limit $U=\infty$. Also very striking is the quasilinear behavior of $n(\omega)$ that is observed for $-V_{b} / 2<\omega<V_{b} / 2$.

Experiments that measure the nonequilibrium distribution function quantity typically use a third (for instance, superconducting) terminal weakly coupled to the system [90-93]. To the best of our knowledge, this quantity has not been measured in quantum dots, and we hope that the present prediction may stimulate some experimental activity.

\section{CONCLUSION: THE FALL OF THE CONVERGENCE WALL}

We have presented a systematic computation of the perturbative expansion of the Anderson impurity model in and out of equilibrium in power of the interaction strength $U$. The main advantage of our Keldysh expansion approach is its ability to calculate directly in the long time steadystate regime. Using our approach, we were able to obtain improved or novel results regarding the nonequilibrium dynamics of strongly interacting quantum dots.

The main contribution of this article lies in the systematic construction of a set of conformal transformations that provide a practical route for a mathematically controlled resummation of series. We have shown how to use analytic conformal transform guided by an approximated location of the singularities of the physical quantities in the $U$ complex plane. We also presented a Bayesian method to control the strong amplification of statistical noise during this procedure, using some simple nonperturbative information. The combination of singularity location, conformal transform crafting, and Bayesian inference provides a robust and generic resummation methodology.

It was noticed recently [77] that, for values of $U$ inside the convergence radius, connected diagrammatic quantum 
Monte Carlo techniques provide a systematic route for calculating the many-body quantum problem in a computational time that only increases polynomially with the requested precision. We argue that the argument in Ref. [77] can be directly extended to systems where the separation hypothesis holds (switching from working with the series in $U$ to the series in $W$ ). We conclude that, in general, systems where the separation hypothesis holds can be computed with a computing time that increases polynomially with the requested precision.

The approach presented here may have implications for a large class of other problems within or beyond condensed matter physics. In particular, a possible extension is to build a real-time (equilibrium or nonequilibrium) quantum impurity solver for DMFT or its extensions or directly address lattice problems such as the Hubbard model. At its core, it consists in techniques to efficiently compute the bare perturbation series and to sum it. Its limitations remain to be explored. They could come from a resurgence of the sign problem, which would manifest itself in a very oscillatory nature of the integrals for expansion coefficients, making them hard to evaluate, or from a difficulty to sum the perturbative series, in particular, for systems with a phase transition, or a non-Fermi-liquid fixed point at a low temperature. In order to address these questions, the technique needs to be applied to more complex models. Work is in progress in this direction.

\section{ACKNOWLEDGMENTS}

The Flatiron Institute is a division of the Simons Foundation. We acknowledge useful discussions with Laura Messio, Volker Meden, and Christophe Mora. We thank our anonymous referee for pointing out Ref. [78]. We acknowledge financial support from the graphene Flagship (ANR FLagera GRANSPORT), the French-U.S. ANR PYRE, and the French-Japan ANR QCONTROL.

\section{APPENDIX A: A TOY MODEL FUNCTION WITH A SINGULARITY ON THE REAL AXIS}

We present here in Fig. 18 a toy model for the resummation of a function $f(U)=1 / \ln [i(1-U)+1]$ that has a pole on the real axis at $U=1$ as well as a branch cut on the curve $U=1-i(1+x)$ with $x \in[0, \infty]$. The aim of this toy model is to show that, even though $f(U)$ has a singularity on the real axis (and, hence, it will be difficult to calculate close to this singularity), it is possible to calculate the function beyond the singularity using a conformal transformation. We use the conformal map $W=$ $[\chi(U)-\chi(0)] /\left[\chi(U)-\chi(0)^{*}\right]$, with $\chi(U)=i \sqrt{(U-1) / p}-i$ that maps the inside of a parabola into the outside of the unit disk (see the upper left and right in Fig. 18). The lower panel in Fig. 18 shows the corresponding resummed series using $N=10,20$, and 30 terms in the expansion of $f(U)$. Although we cannot calculate close to $U=1$, we find that,
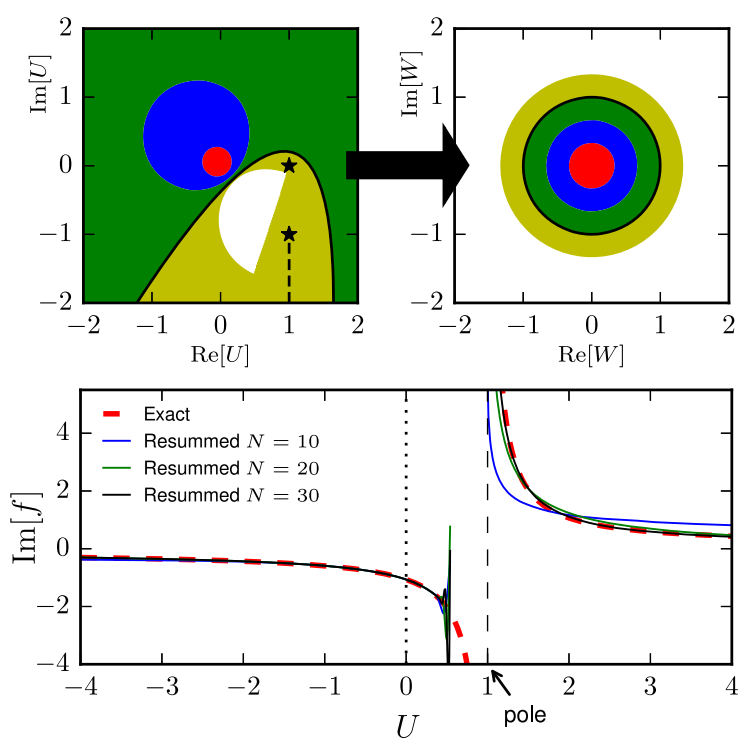

FIG. 18. Resummation of the series of $f(U)=1 / \ln [i(1-U)+1]$ near $U=0$ on the real positive axis, beyond the pole at $U=1 . f$ has a pole at $U=1$ and a branch cut starting at $U=1-i$ and going in a straight line toward $1-i \infty$ (stars and dashed line in top left). We isolate these singularities by applying a conformal map $W=[\chi(U)-\chi(0)] /\left[\chi(U)-\chi(0)^{*}\right]$, with $\chi(U)=$ $i \sqrt{(U-1) / p}-i$. It maps the inside of a parabola into the outside of the unit disk (left and right top). $p$ controls the direction and width of the parabola. Here, $p=0.2 e^{i \pi \times 0.4}$. With $N=30$ terms, one can compute $f$ for all real $U$ (black plain line) except a narrow band around the pole (dashed black vertical line).

with as little as $N=20$ terms in the expansion of $f(U)$, we can recover an accurate description of $f(U)$ for $U>1.2$ from an expansion around $U=0$.

\section{APPENDIX B: CONVERGENCE OF THE PERTURBATION SERIES AT A FINITE TIME}

In this Appendix, we show that, at finite time $t$, the radius of convergence of the perturbation series for the average of an operator $\mathcal{O}$ is infinite, for a system with a finite number of interacting orbitals and an infinite noninteracting bath.

The average value of such an operator is given by

$$
\begin{aligned}
\langle\hat{\mathcal{O}}(t)\rangle & =\left\langle T_{c} e^{-i U \int d u \hat{\mathbf{H}}_{\mathrm{int}}(u)} \hat{\mathcal{O}}(t)\right\rangle \\
& \equiv \sum_{n} \mathcal{O}_{n} \frac{U^{n}}{n !},
\end{aligned}
$$

where the integral goes along the forward-backward Keldysh contour $0 \rightarrow t \rightarrow 0$, the operators are taken in the interaction representation, $T_{c}$ is the usual Keldysh contour ordering operator, and $\hat{\mathbf{H}}_{\text {int }}(u)$ is the interacting part of the Hamiltonian. 
The interaction Hamiltonian has the form

$$
H_{\mathrm{int}}=\sum_{\alpha=1}^{L} V_{\alpha} c_{i(\alpha)}^{\dagger} c_{j(\alpha)}^{\dagger} c_{k(\alpha)} c_{l(\alpha)},
$$

where $1 \leq \alpha \leq L$ labels the interaction terms. The coefficients $\mathcal{O}_{n}$ are obtained from an expression of the form

$$
\begin{aligned}
\mathcal{O}_{n}= & \int_{[0, t]^{n}} d u_{1} \ldots d u_{n} \\
& \times \sum_{a_{i}= \pm 1} \sum_{\alpha_{i}}\left(\prod_{i=1}^{n} a_{i}\right)\left\langle\hat{\mathcal{O}}(t) C_{\left\{a_{i}\right\}\left\{\alpha_{i}\right\}}\left(u_{1}, \ldots, u_{n}\right)\right\rangle,
\end{aligned}
$$

where $C_{\left\{a_{i}\right\}\left\{\alpha_{i}\right\}}\left(u_{1}, \ldots, u_{n}\right)$ is a product of interaction terms, in an order determined by the Keldysh indices $a_{i}$. It has the form

$C_{\left\{a_{i}\right\}\left\{\alpha_{i}\right\}}\left(u_{1}, \ldots, u_{n}\right)=\prod_{p=1}^{n} V_{\alpha_{p}} U_{p}^{\dagger} c_{i\left(\alpha_{p}\right)}^{\dagger} c_{j\left(\alpha_{p}\right)}^{\dagger} c_{k\left(\alpha_{p}\right)} c_{l\left(\alpha_{p}\right)} U_{p}$,

where $U_{p}$ are unitary operators (evolution operator of the noninteracting Hamiltonian). An explicit form of Eq. (B4) can be found in Eq. (12) in Ref. [39].

In the following section of this Appendix, we show the bound

$$
\left|\left\langle\hat{\mathcal{O}}(t) C_{\left\{a_{i}\right\}\left\{\alpha_{i}\right\}}\left(u_{1}, \ldots, u_{n}\right)\right\rangle\right| \leq\|\mathcal{O}\| \bar{V}^{n},
$$

where we denote $\bar{V} \equiv \max _{\alpha}\left|V_{\alpha}\right|$. This bound is obtained for a finite size of the bath (where all operators are finite dimensional matrices and, hence, well defined), but it is independent of the size of the bath and, hence, valid in the thermodynamic limit. We note that the proof of Eq. (B5) does not involve the Wick theorem.

Using Eq. (B5) in Eq. (B4), we arrive at

$$
\left|\mathcal{O}_{n}\right| \leq\|\mathcal{O}\|(2 t \bar{V} L)^{n},
$$

where the factor $2^{n}$ arises from the sum over Keldysh indices and $L^{n}$ from the sum over vertices. Hence, the series (B2) in $U$ is absolutely convergent for all $U$ and has an infinite radius of convergence. Note that this argument is valid, because the electron-electron interaction is present on a finite number of sites only ( $L$ is finite). It would not apply directly to, e.g., the Hubbard model in the thermodynamic limit.

\section{Proof of Eq. (B5)}

We introduce an orthonormal eigenbasis of the noninteracting Hamiltonian (impurity and bath), with eigenvectors $|\xi\rangle$ and eigenvalues $E_{\xi}$ :

$$
\begin{aligned}
\left\langle\hat{\mathcal{O}}(t) C\left(u_{1}, \ldots u_{n}\right)\right\rangle & =\operatorname{Tr}\left(\frac{e^{-\beta H_{0}}}{Z_{0}} \hat{\mathcal{O}}(t) C\left(u_{1}, \ldots u_{n}\right)\right) \\
& =\sum_{\xi} \frac{e^{-\beta E_{\xi}}}{Z_{0}}\left\langle\xi\left|\hat{\mathcal{O}}(t) C\left(u_{1}, \ldots u_{n}\right)\right| \xi\right\rangle .
\end{aligned}
$$

We denote by $\|v\|$ the norm of a vector $|v\rangle$ in the Fock Hilbert space, and we introduce the corresponding induced norm of an operator $\|A\|$ defined as usual by

$$
\|A\| \equiv \sup _{|x\rangle} \frac{\| A|x\rangle \|}{\|x\|} .
$$

Using the short notation $C=C_{\left\{a_{i}\right\}\left\{\alpha_{i}\right\}}\left(u_{1}, \ldots, u_{n}\right)$, the Cauchy-Schwarz inequality, and the definition of the operator norm, we obtain the bound

$$
\begin{aligned}
|\langle\hat{\mathcal{O}}(t) C\rangle| & \leq \sum_{\xi} \frac{e^{-\beta E_{\xi}}}{Z_{0}} \| \hat{\mathcal{O}}(t) C|\xi\rangle\|\| \xi \| \\
& \leq \sum_{\xi} \frac{e^{-\beta E_{\xi}}}{Z_{0}}\|\hat{\mathcal{O}}(t) C\|\|\xi\|^{2} .
\end{aligned}
$$

Using the following properties of the induced operator norm ( $A, B$ being operators):

$$
\|A B\| \leq\|A\|\|B\|,
$$

$$
\left\|U^{\dagger} A U\right\|=\|A\| \text { for any unitary operator } U,
$$

and the fact that $\|\xi\|=1$, we arrive at

$$
|\langle\hat{\mathcal{O}}(t) C\rangle| \leq\|\mathcal{O}\|\|c\|^{4 n} \bar{V}^{n},
$$

where $c$ is a bare creation or destruction operator.

Finally, the crucial point to obtain (B5) is to show that the norm of the $c$ operator is bounded by 1, independently of the size of the bath. Indeed, let us consider a destruction operator $c_{a}$. It acts on the total Fock space (impurity and bath), whose states can be written as

$$
\begin{aligned}
|\psi\rangle= & \sum_{n_{1}, \ldots, n_{K}=0,1} \psi_{0 ; n_{1}, \ldots, n_{K}}\left|0, n_{1}, \ldots, n_{K}\right\rangle \\
& +\psi_{1 ; n_{1}, \ldots, n_{K}}\left|1, n_{1}, \ldots, n_{K}\right\rangle
\end{aligned}
$$

where the first term of the Fock space corresponds to the one-body level $a$ and $n_{i}$ to all the $(K)$ other ones. Then, we have (up to a sign)

$$
c_{a}|\psi\rangle=\sum_{n_{1}, \ldots, n_{K}=0,1} \psi_{1 ; n_{1}, \ldots, n_{K}}\left|0, n_{1}, \ldots, n_{K}\right\rangle
$$

and therefore 


$$
\begin{aligned}
\| c_{a}|\psi\rangle \| & =\sqrt{\sum_{n_{1}, \ldots, n_{K}=0,1}\left|\psi_{1 ; n_{1}, \ldots, n_{K}}\right|^{2}} \\
& \leq \sqrt{\sum_{n_{1}, \ldots, n_{K}=0,1}\left|\psi_{0 ; n_{1}, \ldots, n_{K}}\right|^{2}+\left|\psi_{1 ; n_{1}, \ldots, n_{K}}\right|^{2}}=\|\psi\| .
\end{aligned}
$$

Hence, $\left\|c_{a}\right\| \leq 1$. The same argument can be applied to $\|\mathcal{O}\|$, which concludes the proof of Eq. (B5).

[1] M. Först, C. Manzoni, S. Kaiser, Y. Tomioka, Y. Tokura, R. Merlin, and A. Cavalleri, Nonlinear Phononics as an Ultrafast Route to Lattice Control, Nat. Phys. 7, 854 (2011).

[2] D. Fausti, R. I. Tobey, N. Dean, S. Kaiser, A. Dienst, M. C. Hoffmann, S. Pyon, T. Takayama, H. Takagi, and A. Cavalleri, Light-Induced Superconductivity in a StripeOrdered Cuprate, Science 331, 189 (2011).

[3] D. Nicoletti, E. Casandruc, Y. Laplace, V. Khanna, C. R. Hunt, S. Kaiser, S. S. Dhesi, G. D. Gu, J. P. Hill, and A. Cavalleri, Optically Induced Superconductivity in Striped $\mathrm{La}_{2-x} \mathrm{Ba}_{x} \mathrm{CuO}_{4}$ by Polarization-Selective Excitation in the Near Infrared, Phys. Rev. B 90, 100503(R) (2014).

[4] E. Casandruc, D. Nicoletti, S. Rajasekaran, Y. Laplace, V. Khanna, G. D. Gu, J. P. Hill, and A. Cavalleri, WavelengthDependent Optical Enhancement of Superconducting Interlayer Coupling in $\mathrm{La}_{1.885} \mathrm{Ba}_{0.115} \mathrm{CuO}_{4}$, Phys. Rev. B 91, 174502 (2015).

[5] D. Nicoletti and A. Cavalleri, Nonlinear Light-Matter Interaction at Terahertz, Frequencies, Adv. Opt. Photonics 8, 401 (2016).

[6] D. Nicoletti, D. Fu, O. Mehio, S. Moore, A. S. Disa, G. D. $\mathrm{Gu}$, and A. Cavalleri, Magnetic-Field Tuning of LightInduced Superconductivity in Striped $\mathrm{La}_{2-x} \mathrm{Ba}_{x} \mathrm{CuO}_{4}$, Phys. Rev. Lett. 121, 267003 (2018).

[7] F. Nakamura, M. Sakaki, Y. Yamanaka, S. Tamaru, T. Suzuki, and Y. Maeno, Electric-Field-Induced Metal Maintained by Current of the Mott Insulator $\mathrm{Ca}_{2} \mathrm{RuO}_{4}$, Sci. Rep. 3, 2536 (2013).

[8] J. del Valle, J. G. Ramírez, M. J. Rozenberg, and I. K. Schuller, Challenges in Materials and Devices for Resistive-Switching-Based Neuromorphic Computing, J. Appl. Phys. 124, 211101 (2018).

[9] N. Roch, S. Florens, T. A. Costi, W. Wernsdorfer, and F. Balestro, Observation of the Underscreened Kondo Effect in a Molecular Transistor, Phys. Rev. Lett. 103, 197202 (2009).

[10] J. J. Parks, A. R. Champagne, T. A. Costi, W. W. Shum, A. N. Pasupathy, E. Neuscamman, S. Flores-Torres, P. S. Cornaglia, A. A. Aligia, C. A. Balseiro, G. K.-L. Chan, H. D. Abruña, and D. C. Ralph, Mechanical Control of Spin States in Spin-1 Molecules and the Underscreened Kondo Effect, Science 328, 1370 (2010).

[11] Z. Iftikhar, S. Jezouin, A. Anthore, U. Gennser, F. D. Parmentier, A. Cavanna, and F. Pierre, Two-Channel Kondo Effect and Renormalization Flow with Macroscopic Quantum Charge States, Nature (London) 526, 233 (2015).

[12] Z. Iftikhar, A. Anthore, A. K. Mitchell, F. D. Parmentier, U. Gennser, A. Ouerghi, A. Cavanna, C. Mora, P. Simon, and
F. Pierre, Tunable Quantum Criticality and Super-ballistic Transport in a "Charge" Kondo Circuit, Science 360, 1315 (2018).

[13] T. Giamarchi, Quantum Physics in One Dimension, International Series of Monographs in Physics (Clarendon, Oxford, 2004).

[14] K. J. Thomas, J. T. Nicholls, M. Y. Simmons, M. Pepper, D. R. Mace, and D. A. Ritchie, Possible Spin Polarization in a One-Dimensional Electron Gas, Phys. Rev. Lett. 77, 135 (1996).

[15] K. J. Thomas, J. T. Nicholls, N. J. Appleyard, M. Y. Simmons, M. Pepper, D. R. Mace, W. R. Tribe, and D. A. Ritchie, Interaction Effects in a One-Dimensional Constriction, Phys. Rev. B 58, 4846 (1998).

[16] A.P. Micolich, What Lurks below the Last Plateau: Experimental Studies of the $0.7 \times 2 e^{2} / h$ Conductance Anomaly in One-Dimensional Systems, J. Phys. Condens. Matter 23, 443201 (2011).

[17] J. Preskill, Quantum Computing in the NISQ Era and Beyond, Quantum 2, 79 (2018).

[18] F. Reininghaus, M. Pletyukhov, and H. Schoeller, Kondo Model in Nonequilibrium: Interplay between Voltage, Temperature, and Crossover from Weak to Strong Coupling, Phys. Rev. B 90, 085121 (2014).

[19] F. Schwarz, I. Weymann, J. von Delft, and A. Weichselbaum, Nonequilibrium Steady-State Transport in Quantum Impurity Models: A Thermofield and Quantum Quench Approach Using Matrix Product States, Phys. Rev. Lett. 121, 137702 (2018).

[20] T. Fujii and K. Ueda, Perturbative Approach to the Nonequilibrium Kondo Effect in a Quantum Dot, Phys. Rev. B 68, 155310 (2003).

[21] R. Van Roermund, S.-y. Shiau, and M. Lavagna, Anderson Model out of Equilibrium: Decoherence Effects in Transport through a Quantum Dot, Phys. Rev. B 81, 165115 (2010).

[22] N. S. Wingreen and Y. Meir, Anderson Model out of Equilibrium: Noncrossing-Approximation Approach to Transport through a Quantum Dot, Phys. Rev. B 49, 11040 (1994).

[23] S. R. White, Density Matrix Formulation for Quantum Renormalization Groups, Phys. Rev. Lett. 69, 2863 (1992).

[24] S. R. White, Density-Matrix Algorithms for Quantum Renormalization Groups, Phys. Rev. B 48, 10345 (1993).

[25] U. Schollwöck, The Density-Matrix Renormalization Group, Rev. Mod. Phys. 77, 259 (2005).

[26] F. B. Anders and A. Schiller, Real-Time Dynamics in Quantum-Impurity Systems: A Time-Dependent Numerical Renormalization-Group Approach, Phys. Rev. Lett. 95, 196801 (2005).

[27] F. Heidrich-Meisner, A. E. Feiguin, and E. Dagotto, RealTime Simulations of Nonequilibrium Transport in the SingleImpurity Anderson Model, Phys. Rev. B 79, 235336 (2009).

[28] J. Eckel, F. Heidrich-Meisner, S. G. Jakobs, M. Thorwart, M. Pletyukhov, and R. Egger, Comparative Study of Theoretical Methods for Non-Equilibrium Quantum Transport, New J. Phys. 12, 043042 (2010).

[29] L. Mühlbacher and E. Rabani, Real-Time Path Integral Approach to Nonequilibrium Many-Body Quantum Systems, Phys. Rev. Lett. 100, 176403 (2008). 
[30] P. Werner, T. Oka, and A. J. Millis, Diagrammatic Monte Carlo Simulation of Nonequilibrium Systems, Phys. Rev. B 79, 035320 (2009).

[31] P. Werner, T. Oka, M. Eckstein, and A. J. Millis, WeakCoupling Quantum Monte Carlo Calculations on the Keldysh Contour: Theory and Application to the CurrentVoltage Characteristics of the Anderson Model, Phys. Rev. B 81, 035108 (2010).

[32] M. Schiró and M. Fabrizio, Real-Time Diagrammatic Monte Carlo for Nonequilibrium Quantum Transport, Phys. Rev. B 79, 153302 (2009).

[33] M. Schiró, Real-Time Dynamics in Quantum Impurity Models with Diagrammatic Monte Carlo, Phys. Rev. B 81, 085126 (2010).

[34] G. Cohen, D. R. Reichman, A. J. Millis, and E. Gull, Green's Functions from Real-Time Bold-Line Monte Carlo, Phys. Rev. B 89, 115139 (2014).

[35] G. Cohen, E. Gull, D. R. Reichman, and A. J. Millis, Green's Functions from Real-Time Bold-Line Monte Carlo Calculations: Spectral Properties of the Nonequilibrium Anderson Impurity Model, Phys. Rev. Lett. 112, 146802 (2014).

[36] G. Cohen, E. Gull, D. R. Reichman, and A. J. Millis, Taming the Dynamical Sign Problem in Real-Time Evolution of Quantum Many-Body Problems, Phys. Rev. Lett. 115, 266802 (2015).

[37] H.-T. Chen, G. Cohen, and D. R. Reichman, Inchworm Monte Carlo for Exact Non-adiabatic Dynamics. I. Theory and Algorithms, J. Chem. Phys. 146, 054105 (2017).

[38] H.-T. Chen, G. Cohen, and D. R. Reichman, Inchworm Monte Carlo for Exact Non-adiabatic Dynamics. II. Benchmarks and Comparison with Established Methods, J. Chem. Phys. 146, 054106 (2017).

[39] R. E. V. Profumo, C. Groth, L. Messio, O. Parcollet, and X. Waintal, Quantum Monte Carlo for Correlated Out-ofEquilibrium Nanoelectronic Devices, Phys. Rev. B 91, 245154 (2015).

[40] A. Georges, G. Kotliar, W. Krauth, and M. J. Rozenberg, Dynamical Mean-Field Theory of Strongly Correlated Fermion Systems and the Limit of Infinite Dimensions, Rev. Mod. Phys. 68, 13 (1996).

[41] G. Kotliar, S. Y. Savrasov, K. Haule, V. S. Oudovenko, O. Parcollet, and C. A. Marianetti, Electronic Structure Calculations with Dynamical Mean-Field Theory, Rev. Mod. Phys. 78, 865 (2006).

[42] H. Aoki, N. Tsuji, M. Eckstein, M. Kollar, T. Oka, and P. Werner, Nonequilibrium Dynamical Mean-Field Theory and Its Applications, Rev. Mod. Phys. 86, 779 (2014).

[43] J. Rammer, Quantum Field Theory of Non-equilibrium States (Cambridge University Press, Cambridge, England, 2007).

[44] N. V. Prokof'ev and B. V. Svistunov, Polaron Problem by Diagrammatic Quantum Monte Carlo, Phys. Rev. Lett. 81, 2514 (1998).

[45] A. S. Mishchenko, N. V. Prokof'ev, A. Sakamoto, and B. V. Svistunov, Diagrammatic Quantum Monte Carlo Study of the Fröhlich Polaron, Phys. Rev. B 62, 6317 (2000).

[46] K. Van Houcke, E. Kozik, N. Prokof'ev, and B. Svistunov, Diagrammatic Monte Carlo, Phys. Procedia 6, 95 (2010).
[47] N. Prokof'ev and B. Svistunov, Bold Diagrammatic Monte Carlo Technique: When the Sign Problem Is Welcome, Phys. Rev. Lett. 99, 250201 (2007).

[48] N. V. Prokof'ev and B. V. Svistunov, Bold Diagrammatic Monte Carlo: A Generic Sign-Problem Tolerant Technique for Polaron Models and Possibly Interacting Many-Body Problems, Phys. Rev. B 77, 125101 (2008).

[49] E. Gull, D. R. Reichman, and A. J. Millis, Bold-Line Diagrammatic Monte Carlo Method: General Formulation and Application to Expansion around the Noncrossing Approximation, Phys. Rev. B 82, 075109 (2010).

[50] E. Kozik, K. Van Houcke, E. Gull, L. Pollet, N. Prokof'ev, B. Svistunov, and M. Troyer, Diagrammatic Monte Carlo for Correlated Fermions, Europhys. Lett. 90, 10004 (2010).

[51] L. Pollet, Recent Developments in Quantum Monte Carlo Simulations with Applications for Cold Gases, Rep. Prog. Phys. 75, 094501 (2012).

[52] K. Van Houcke, F. Werner, E. Kozik, N. Prokof'ev, B. Svistunov, M. J. H. Ku, A. T. Sommer, L. W. Cheuk, A. Schirotzek, and M. W. Zwierlein, Feynman Diagrams versus Fermi-Gas Feynman Emulator, Nat. Phys. 8, 366 (2012).

[53] S. A. Kulagin, N. Prokof'ev, O. A. Starykh, B. Svistunov, and C. N. Varney, Bold Diagrammatic Monte Carlo Method Applied to Fermionized Frustrated Spins, Phys. Rev. Lett. 110, 070601 (2013).

[54] S. A. Kulagin, N. Prokof'ev, O. A. Starykh, B. Svistunov, and C. N. Varney, Bold Diagrammatic Monte Carlo Technique for Frustrated Spin Systems, Phys. Rev. B 87, 024407 (2013).

[55] J. Gukelberger, E. Kozik, L. Pollet, N. Prokof'ev, M. Sigrist, B. Svistunov, and M. Troyer, p-Wave Superfluidity by SpinNematic Fermi Surface Deformation, Phys. Rev. Lett. 113, 195301 (2014).

[56] Y. Deng, E. Kozik, N. V. Prokof'ev, and B. V. Svistunov, Emergent BCS Regime of the Two-Dimensional Fermionic Hubbard Model: Ground-State Phase Diagram, Europhys. Lett. 110, 57001 (2015).

[57] Y. Huang, K. Chen, Y. Deng, N. Prokof'ev, and B. Svistunov, Spin-Ice State of the Quantum Heisenberg Antiferromagnet on the Pyrochlore Lattice, Phys. Rev. Lett. 116, 177203 (2016).

[58] R. Rossi, T. Ohgoe, E. Kozik, N. Prokof'ev, B. Svistunov, K. Van Houcke, and F. Werner, Contact and Momentum Distribution of the Unitary Fermi Gas, Phys. Rev. Lett. 121, 130406 (2018).

[59] K. Van Houcke, F. Werner, T. Ohgoe, N. V. Prokof'ev, and B. V. Svistunov, Diagrammatic Monte Carlo Algorithm for the Resonant Fermi Gas, Phys. Rev. B 99, 035140 (2019).

[60] R. Rossi, Determinant Diagrammatic Monte Carlo Algorithm in the Thermodynamic Limit, Phys. Rev. Lett. 119, 045701 (2017).

[61] A. Moutenet, W. Wu, and M. Ferrero, Determinant Monte Carlo Algorithms for Dynamical Quantities in Fermionic Systems, Phys. Rev. B 97, 085117 (2018).

[62] F. Simkovic IV and E. Kozik, Determinant Monte Carlo for Irreducible Feynman Diagrams in the Strongly Correlated Regime, Phys. Rev. B 100, 121102(R) (2019).

[63] R. Rossi, Direct Sampling of the Self-Energy with Connected Determinant Monte Carlo, arXiv:1802.04743. 
[64] C. Bertrand, O. Parcollet, A. Maillard, and X. Waintal, Quantum Monte Carlo Algorithm for Out-of-Equilibrium Green's Functions at Long Times, Phys. Rev. B 100, 125129 (2019).

[65] O. Parcollet, M. Ferrero, T. Ayral, H. Hafermann, I. Krivenko, L. Messio, and P. Seth, TRIQS: A Toolbox for Research on Interacting Quantum Systems, Comput. Phys. Commun. 196, 398 (2015).

[66] A. C. Hewson, The Kondo Problem to Heavy Fermions, Cambridge Studies in Magnetism (Cambridge University Press, Cambridge, England, 1993).

[67] G. Stefanucci and R. van Leeuwen, Nonequilibrium ManyBody Theory of Quantum Systems: A Modern Introduction (Cambridge University Press, Cambridge, England, 2013).

[68] Y. Meir and N. S. Wingreen, Landauer Formula for the Current through an Interacting Electron Region, Phys. Rev. Lett. 68, 2512 (1992).

[69] G. H. Hardy, Divergent Series (Oxford University, New York, 1949).

[70] G. A. Baker and P. Graves-Morris, Padé Approximants, Encyclopedia of Mathematics (Cambridge University Press, Cambridge, England, 1996).

[71] E. Lindelöf, Le Calcul des Résidus et ses Applications à la Théorie des Fonctions (Gauthier-Villars, Paris, 1905).

[72] T. Ayral and O. Parcollet, Mott Physics and Spin Fluctuations: A Unified Framework, Phys. Rev. B 92, 115109 (2015).

[73] T. Ayral, J. Vučičević, and O. Parcollet, Fierz Convergence Criterion: A Controlled Approach to Strongly Interacting Systems with Small Embedded Clusters, Phys. Rev. Lett. 119, 166401 (2017).

[74] R. Rossi, T. Ohgoe, K. Van Houcke, and F. Werner, Resummation of Diagrammatic Series with Zero Convergence Radius for Strongly Correlated Fermions, Phys. Rev. Lett. 121, 130405 (2018).

[75] A. J. Guttmann, in Asymptotic Analysis of Power-Series Expansions, Phase Transitions and Critical Phenomena Vol. 13, edited by C. Domb and J. Lebowitz (Academic, New York, 1989).

[76] T. A. Driscoll and L. N. Trefethen, Schwarz-Christoffel Mapping, Cambridge Monographs on Applied and Computational Mathematics (Cambridge University Press, Cambridge, England, 2002).

[77] R. Rossi, N. Prokof'ev, B. Svistunov, K. Van Houcke, and F. Werner, Polynomial Complexity Despite the Fermionic Sign, Europhys. Lett. 118, 10004 (2017).

[78] B. Horvatić and V. Zlatić, Equivalence of the Perturbative and Bethe-Ansatz. Solution of the Symmetric Anderson Hamiltonian, J. Phys. (Paris) 46, 1459 (1985).

[79] V. Zlatić and B. Horvatić, Series Expansion for the Symmetric Anderson Hamiltonian, Phys. Rev. B 28, 6904 (1983).
[80] H. Theil, A Rank-Invariant Method of Linear and Polynomial Regression Analysis, in Henri Theil's Contributions to Economics and Econometrics: Econometric Theory and Methodology, edited by B. Raj and J. Koerts (Springer Netherlands, Dordrecht, 1992), pp. 345-381.

[81] P. Nordlander, M. Pustilnik, Y. Meir, N. S. Wingreen, and D. C. Langreth, How Long Does It Take for the Kondo Effect to Develop?, Phys. Rev. Lett. 83, 808 (1999).

[82] R. Bulla, T. A. Costi, and T. Pruschke, Numerical Renormalization Group Method for Quantum Impurity Systems, Rev. Mod. Phys. 80, 395 (2008).

[83] W. Hofstetter, Generalized Numerical Renormalization Group for Dynamical Quantities, Phys. Rev. Lett. 85, 1508 (2000).

[84] A. I. Tóth, C. P. Moca, Ö. Legeza, and G. Zaránd, Density Matrix Numerical Renormalization Group for Non-Abelian Symmetries, Phys. Rev. B 78, 245109 (2008).

[85] R. Bulla, A. C. Hewson, and T. Pruschke, Numerical Renormalization Group Calculations for the Self-Energy of the Impurity Anderson Model, J. Phys. Condens. Matter 10, 8365 (1998).

[86] See Supplemental Material at http://link.aps.org/ supplemental/10.1103/PhysRevX.9.041008 for power series and one-sigma error bars for the retarded and/or lesser Green's function of several Anderson impurity models (at or out of equilibrium) studied. Content of each file is described in its first line (header). Each line contains the series for a given frequency.

[87] S. Hershfield, J. H. Davies, and J. W. Wilkins, Probing the Kondo Resonance by Resonant Tunneling through an Anderson Impurity, Phys. Rev. Lett. 67, 3720 (1991).

[88] S. Hershfield, J. H. Davies, and J. W. Wilkins, Resonant Tunneling through an Anderson Impurity. I. Current in the Symmetric Model, Phys. Rev. B 46, 7046 (1992).

[89] F. B. Anders, Steady-State Currents through Nanodevices: A Scattering-States Numerical Renormalization-Group Approach to Open Quantum Systems, Phys. Rev. Lett. 101, 066804 (2008).

[90] H. Pothier, S. Guéron, N. O. Birge, D. Esteve, and M. H. Devoret, Energy Distribution Function of Quasiparticles in Mesoscopic Wires, Phys. Rev. Lett. 79, 3490 (1997).

[91] A. Anthore, F. Pierre, H. Pothier, and D. Esteve, MagneticField-Dependent Quasiparticle Energy Relaxation in Mesoscopic Wires, Phys. Rev. Lett. 90, 076806 (2003).

[92] B. Huard, A. Anthore, F. Pierre, H. Pothier, N. O. Birge, and D. Esteve, Intensity of Coulomb Interaction between Quasiparticles in Diffusive Metallic Wires, Solid State Commun. 131, 599 (2004).

[93] Y.-F. Chen, T. Dirks, G. Al-Zoubi, N. O. Birge, and N. Mason, Nonequilibrium Tunneling Spectroscopy in Carbon Nanotubes, Phys. Rev. Lett. 102, 036804 (2009). 